OPEN ACCESS

Edited by:

Yiliao Song,

University of Technology Sydney,

Australia

Reviewed by:

Tong Niu,

Zhengzhou University, China

Danxiang Wei,

Macau University of Science and

Technology, China

*Correspondence:

Hongmin $\mathrm{Li}$

Ihm@nefu.edu.ch

Specialty section:

This article was submitted to

Environmental Informatics and Remote

Sensing,

a section of the journal

Frontiers in Environmental Science

Received: 11 December 2021

Accepted: 17 January 2022

Published: 25 February 2022

Citation:

Bai L, Li H, Zeng B and Huang X (2022)

Design of a Combined System Based on Multi-Objective Optimization for

Fine Particulate Matter

(PM 2.5) Prediction.

Front. Environ. Sci. 10:833374.

doi: 10.3389/fenvs.2022.833374

\section{Design of a Combined System Based on Multi-Objective Optimization for Fine Particulate Matter $\left(\mathbf{P M}_{2.5}\right)$ Prediction}

\author{
Lu Bai ${ }^{1}$, Hongmin $L^{2 *}$, Bo Zeng ${ }^{3}$ and Xiaojia Huang ${ }^{4}$ \\ ${ }^{1}$ Department of Mathematics, University of Macau, Macau, China, ${ }^{2}$ College of Economics and Management, Northeast Forestry \\ University, Harbin, China, ${ }^{3}$ Collaborative Innovation Center for Chongqing's Modern Trade Logistics and Supply Chain, \\ Chongqing Technology and Business University, Chongqing, China, ${ }^{4}$ School of Information Science and Engineering, Lanzhou \\ University, Lanzhou, China
}

Air pollution forecasting plays a pivotal role in environmental governance, so a large number of scholars have devoted themselves to the study of air pollution forecasting models. Although numerous studies have focused on this field, they failed to consider fully the linear feature, non-linear feature, and fuzzy features contained in the original series. To fill this gap, a new combined system is built to consider features in the original series and accurately forecast $\mathrm{PM}_{2.5}$ concentration, which incorporates an efficient data decomposition strategy to extract the primary features of the $\mathrm{PM}_{2.5}$ concentration series and remove the noise component, and five forecasting models selected from three types of models to obtain the preliminary forecasting results, and a multi-objective optimization algorithm to combine the prediction results to produce the final prediction values. Empirical studies results indicated that in terms of RMSE the developed combined system achieves $0.6526 \%, 0.8101 \%$, and $0.7750 \%$ in three study cities, respectively. Compared to other prediction models, the RMSE improved by $60 \%$ on average in the study cities.

Keywords: combined forecasting model, air pollution forecasting, improved extreme learning machine, data decomposition, multi-objective optimization approach, fuzzy computation and forecasting

\section{INTRODUCTION}

Atmosphere pollutants can cause a variety of diseases (Organization, 2014, March 25; Glencross et al., 2020), and cause other environmental problems (Grennfelt et al., 2020; Manisalidis et al., 2020), endangering human survival. To alleviate the impacts of atmosphere pollution, support environmental management, more scholars are focusing on air pollution forecasting.

Air pollution forecasting is a complex task since there are multiple influences on pollutant concentrations, such as weather, wind speed and direction, geographic location, pollution emission and absorption, and policies, etc. Therefore, the concentration series are chaotic and usually contain both linear and non-linear features (Niska et al., 2004). In the past decades, the forecasting of air 
pollution has attracted wide academic interest, and much effort has been made to forecast concentration using various approaches. Generally speaking, these approaches can be divided into four categories: individual models, hybrid models, combined models, and meteorological models. The meteorological models are based on the physical and chemical processes of pollutants in the atmosphere. This type of model is the subject of atmospheric research. For individual methods, the concentration series are modeled and forecast by one type of model, such as the traditional statistical model, Auto-Regressive Integrated Moving-Average (ARima), neuron network model, Back-propagation Neural Network (BPnn), etc. Research on this type of model was mainly concentrated before 2010. Such as Niska et al. (2004) used a parallel genetic algorithm to select the inputs for the multi-layer perceptron model to forecast hourly concentrations of nitrogen dioxide. Goyal et al. (2006) compared the performance of three statistical models for forecasting the concentration of respirable suspended particulate matter. These three models are multiple linear regression, ARima, and the combination of ARima and Multiple linear regression. The prediction results show that the combination of ARima and Multiple linear regression performs better. Kurt et al. (2008) built an online forecasting system by utilizing BPnn to predict the concentrations of $\mathrm{SO}_{2}, \mathrm{PM}_{10}$ and $\mathrm{CO}$.

With the development of forecasting methods, a new type of forecasting method, the hybrid model, has been proposed and widely used. The hybrid models can advance forecasting by combining different forecasting techniques, such as combining statistical models and machine learning methods. This combination can compensate for the limitations of individual methods by taking advantage of different methods. Zhu et al. (2017) decomposed the original data into several intrinsic mode functions (IMFs, containing the important information) and noise series. Then, they built two hybrid forecasting models to forecast the daily air quality index, including least square support vector regression, Holt-Winters additive model, Grey model, and seasonal ARima. By combining the Hampel identifier, empirical wavelet transform, Elman neural network, and Outlier-robust extreme learning machine, a novel hybrid algorithm was proposed in (Liu et al., 2019), which improved the forecasting accuracy of fine particle concentrations. Similarly, using a data preprocessing module and an optimal forecasting module, Wang et al. (2020a) proposed a new well-performing hybrid model to forecast daily air quality, which combines Hampel identifier, Variational mode decomposition, Sine cosine algorithm, and Extreme learning machine to forecast daily air quality.

With the development of different forecasting techniques, combined forecasting has gradually become the research focus of scholars. The main idea of the combined models is to combine the forecasting results of several individual models. Yang et al. (2020) proposed a combined forecasting system combining Complementary ensemble empirical mode decomposition (CEEMD), BPnn, Extreme learning machine, and Double Exponential Smoothing, then used fuzzy theory and Cuckoo search algorithm to determine aggregation weights to obtain final results. Based on the wavelet transform and neural networks, Liu et al. (2021) constructed a new combined model. In their study, discrete Wavelet transform was used to decompose the $\mathrm{NO}_{2}$ concentration series. Next to the Long shortterm memory neural network (LSTM), Gated recurrent units and Bi-directional LSTM were utilized to forecast $\mathrm{NO}_{2}$ concentration. Finally, they applied two numerical weighting methods combining the three single forecasting results.

However, these forecasting models have various problems. Because of their simple structure and convenient calculation, statistical models have been widely used, but the linear mapping and poor extrapolation limit the forecasting performance of such models (Wang et al., 2020c). Artificial intelligence methods are widely used own to their strong learning ability and ability to handle nonlinear features, but such methods tend to fall into local optima and overfitting. Moreover, their performance is dependent on artificially set hyperparameters (Niu and Wang, 2019). To avoid the defects of the individual models, several hybrid models have been developed. However, hybrid models still do not always perform best using only one single predictor, since the single model cannot capture various features contained in the series (Yang et al., 2020). Therefore, the combination models gradually developed. However, previous combined models usually combine a certain type of model. This combination can only continuously extract one type of feature in the series, and still cannot analyze the multiple features contained in the series. This paper summarizes the above-mentioned types of models in Table 1. To fill this gap, a novel combined model containing a data decomposition module, a forecasting module consisting of different types of forecasting models, and a combination module weighted by multi-objective optimization algorithms is proposed in this paper. More specifically, the complete ensemble empirical mode decomposition with adaptive noise (cEEMDan) strategy is used for data decomposition to reduce the influence of the noise in the original series. Whereafter, five predictors from three types of models are introduced to construct the forecasting module. These five predictors are one statistical model, three neuron networks, and a hesitant fuzzy forecasting model. The multi-optimization algorithm is utilized to aggregate the forecasting results of five individual models to obtain the final forecasting results.

Based on the above content, the main contributions and innovations of this research are summarized as follows:

1) A novel combined forecasting system is proposed by combining with data decomposition strategy, forecasting models, and multi-objective optimization algorithm. To obtain better forecasting performance, the strategy of "decomposition and ensemble" is introduced to capture different features and remove the noise of the original data, five individual models are used to forecast the decomposed data, and a multi-objective optimization algorithm is utilized to obtain the optimal weights of individual models and integrate them. The empirical experiments demonstrated that the proposed combined forecasting system can provide accurate prediction results for $\mathrm{PM}_{2.5}$ concentration forecasting, and can provide data support for decision-making. 
TABLE 1 | Summary of the different types of models.

\begin{tabular}{|c|c|c|c|c|c|}
\hline Models & References & Variables & Results & Advantages & Dis-advantages \\
\hline \multicolumn{6}{|c|}{ Meteorological Models } \\
\hline ADMS-Urban & $\begin{array}{l}\text { Dédelè and } \\
\text { Miškinytė } \\
\text { (2019) }\end{array}$ & $\mathrm{PM}_{10}$ & $\begin{array}{l}\text { According to the analysis of } \mathrm{PM}_{10} \text { in } \\
\text { the study cities, the ADMS-Urban } \\
\text { model takes into account the different } \\
\text { characteristics of the sites and can be } \\
\text { applied to the exposure estimates in } \\
\text { the cohort studies }\end{array}$ & & \\
\hline AERMOD & $\begin{array}{l}\text { Mousavi et al. } \\
\text { (2021) }\end{array}$ & $\begin{array}{l}\mathrm{CO} \\
\mathrm{CO}_{2}, \mathrm{SO}_{2}\end{array}$ & $\begin{array}{l}\text { According to the experimental } \\
\text { results, the } \mathrm{CO} \text { concentration of } 8 \mathrm{~h} \\
\text { and the } \mathrm{SO}_{2} \text { concentration of } 1 \mathrm{~h} \text { in } \\
\text { the cold season may aggravate the } \\
\text { impact on the breathing air of } \\
\text { residents around the studied refinery }\end{array}$ & $\begin{array}{l}\text { No historical weather data is } \\
\text { required, the accuracy is high, and } \\
\text { the causal relationship between the } \\
\text { input and output in the model is } \\
\text { clear, which makes the model more } \\
\text { readable }\end{array}$ & $\begin{array}{l}\text { The models are very computationally } \\
\text { intensive and time-consuming, and } \\
\text { the quality of the input data has a } \\
\text { significant impact on the prediction } \\
\text { results, as even small data deviations } \\
\text { can lead to large differences in the } \\
\text { results }\end{array}$ \\
\hline $\begin{array}{l}\text { CRTM and WRF- } \\
\text { Chem }\end{array}$ & $\begin{array}{l}\text { Cheng et al. } \\
(2019)\end{array}$ & $\mathrm{PM}_{10}$ & $\begin{array}{l}\text { The results show that assimilation of } \\
\text { Lidar data can effectively improve the } \\
\text { prediction effect. The predicted } \mathrm{PM}_{2.5} \\
\text { concentration of the constructed } \\
\text { model is closer to the observed value, } \\
\text { and the low deviation of the model is } \\
\text { significantly reduced }\end{array}$ & & \\
\hline
\end{tabular}

Statistical models

\begin{tabular}{|c|c|c|c|c|c|}
\hline ARima & $\begin{array}{l}\text { Zhang et al. } \\
\text { (2018) }\end{array}$ & $\mathrm{PM}_{2.5}$ & $\begin{array}{l}\text { The trend of fluctuations in PM2.5 } \\
\text { concentrations in the forecast period } \\
\text { is similar to the trend in the first two of } \\
\text { the forecast period, which is a } \\
\text { seasonal fluctuation }\end{array}$ & $\begin{array}{l}\text { The structure of the statistical } \\
\text { model is simple, so it is easy to } \\
\text { implement and easy to calculate }\end{array}$ & $\begin{array}{l}\text { This kind of models need a large } \\
\text { amount of historical data. The } \\
\text { statistical models cannot analyze non- } \\
\text { linear series, and have poor } \\
\text { extrapolation }\end{array}$ \\
\hline $\begin{array}{l}\text { MLR and ARima } \\
\text { and MLR-ARima }\end{array}$ & $\begin{array}{l}\text { Goyal et al. } \\
(2006)\end{array}$ & PM & $\begin{array}{l}\text { According to the experimental } \\
\text { results, the prediction performance of } \\
\text { the combination of ARima and } \\
\text { multiple regression is better }\end{array}$ & & \\
\hline
\end{tabular}

\section{Neuron networks}

MLP and GA Niska et al. $\quad \mathrm{NO}_{2}$ (2004)

BPnn
Kurt et al. (2008) $\quad \mathrm{SO}_{2}$

$\mathrm{PM}_{10}, \mathrm{CO}$
The results show that the GA is able to reduce computation by eliminating irrelevant inputs and search for feasible high-level architectures
The neuron networks have strong learning ability and can handle nonlinear features in the data
This kind of models need a large amount of historical data. And may fall into the local optima and overfitting. Moreover, their performance is dependent on artificially set hyperparameters

Experiments show that quite accurate predictions of air pollutant indicator levels are possible with proposed online air pollution forecasting system

\section{Hybrid models}

\begin{tabular}{lll}
\hline EMD-SVR- & Zhu et al. (2017) & AQI \\
SARima and & & \\
EMD-HW/GM- & & \\
SARima & & \\
HI-IEWT-Enn- & Liu et al. (2019) & PM $_{2.5}$ \\
ORelm & &
\end{tabular}

ORelm

$\begin{array}{ll}\text { HI-VMD- } & \text { Wang et al. } \\ \text { SCA-ELM } & \text { (2020a) }\end{array}$

The proposed hybrid model can be used as an effective and simple tool for air pollution early warning and management, and can be applied to predict other pollution indices The performance of the proposed model is improved in multi-step forecasting, while the reconstruction method solves the overfitting problem and improves the stability of the hybrid model The proposed hybrid model gives a new feasible method for air pollution forecasting, which is beneficial to air quality management
Hybrid models can integrate the advantages of individual models, so that the forecasting more accuracy
This kind of models not always perform best using only one type of models, since they cannot capture various features contained in the series 
2) Three types of forecasting models are introduced to establish the robust forecasting module. In order to fully analyze the various features contained in the series, three types of forecasting models are combined. Since there are multiple influences on air pollution, the pollutant concentration series are chaotic and usually contain linear and non-linear features. The utilized three different types of models can analyze different features in the series, the statistical model can deal with linear features, neuron networks can cope with non-linear features, and the hesitant fuzzy forecasting model is used to analyze the fuzzy features. This ensures the diversity of the system and avoids that the combined model focuses on a certain type of specific model while ignoring other features in the series.

3) A multi-objective optimization algorithm is used to weight the individual forecasting models. In this study, the final forecasting results are equal to the weighted sum of individual model forecasting results, so the weight of each model is a key to ensuring forecasting accuracy. Most previous studies used numerical weighting methods, so this paper compares several numerical weighting methods with optimization algorithms. In addition, the idea of some feature selection methods can also be regarded as a kind of weighting, so this paper also chooses two feature selection methods, Max-Relevance and Min-Redundancy (MRMR) and ReliefF, as weighting methods to participate in the comparison of weighting methods. However, after the comparison in this study, the multi-objective optimization algorithm is proven to be the best weighting method, outperforming not only numerical methods but also feature selection methods.

For the convenience of the readers, all abbreviation words are listed in Table 2. The remainder of this paper is organized as follows: the basic methodology of utilized methods and the system design is introduced in Section 2. The experimental design, the experiment results, and the analysis of the results are presented in Section 3. The significance test and stability test are discussed in Section 4. Finally, Section 5 provides the conclusion of this study.

\section{FRAMEWORK OF THE DEVELOPED COMBINED FORECASTING SYSTEM}

In this section, the utilized methodologies of the combined system are introduced. These methodologies include the cEEMDan, ARima, BPnn, $\ell_{2,1}$-norm and Random Fourier Mapping-Based Extreme Learning Machine $\left(\ell_{2,1}\right.$ RFelm), Echo state network (ESn), Fuzzy time series forecasting based on hesitant fuzzy sets (HFs) and Multi-objective salp swarm algorithm (mSSa).

\subsection{Data Decomposition}

Due to various factors, the monitoring data, especially the air pollutant concentration data, will have fluctuations and noise, which will affect the further analysis of the data. Therefore, to extract the characteristics of the series, cEEMDan is used to decompose the original series.

cEEMDan is an improved method based on the Empirical Mode Decomposition (EMD) method, which adds adaptive noise series at each stage of the EMD decomposition to make the decomposition more perfect while avoiding mode mixing problem (Torres et al., 2011). EMD-series methods can decompose any complicated series into a finite of intrinsic mode functions (IMFs), and each IMF represents the implicit characteristics of the original series.

The decomposition results of EMD are some IMFs and residuals, and the decomposition process is the process of finding the IMFs. Assume the original $\mathrm{PM}_{2.5}$ concentration series $X(t), t=1, \ldots, n$ is decomposed into $k$ IMFs, the EMD process can be summarized as follows:

Let $\boldsymbol{a}_{\boldsymbol{0}}(\boldsymbol{t})=X(t)$ be the signal being analyzed, find all the local maximum and minimum of $a_{0}$, and interpolate to form upper and lower envelopes, denoted as $\boldsymbol{a}_{0}^{\text {max }}$ and $\boldsymbol{a}_{0}^{\text {min }}$, respectively. Calculate the mean of upper and lower envelopes as $m_{11}(t)=\left[\boldsymbol{a}_{\mathbf{0}}^{\max }(\boldsymbol{t})+\boldsymbol{a}_{\mathbf{0}}^{\min }(\boldsymbol{t})\right] / 2$. Next, extract the first detailed component as $\overline{\bar{\chi}}_{11}(t)=\boldsymbol{a}_{0}(\boldsymbol{t})-m_{11}(t)$. If $\overline{\bar{\chi}}_{11}(t)$ satisfies the two conditions of IMFs, $\overline{\bar{\chi}}_{11}(t)$ is the first IMF, denoted as IMF 1 ; else, $\overline{\bar{\chi}}_{11}(t)$ is considered as the signal, and repeat the Step 1-Step 3 until the decomposition result $\overline{\bar{\chi}}_{1 j}$ satisfies the conditions at $j$-th decomposition, $\operatorname{IMF}_{\mathbf{1}}(\mathbf{t})=\mathbf{a}_{\mathbf{0}}(\mathbf{t})-\sum_{i=1}^{r} m_{1 j}(t)$. And the first residue is $\hat{\gamma}_{1}(t)=\sum_{i=1}^{r} m_{1 j}(t)$. Set $\hat{\gamma}_{1}$ as the signal to be decomposed, and keep repeating the Step 1-Step 4 until the final residual $\hat{\gamma}_{k}$ becomes a monotonic function. At the end of this decomposition, the original series can be represented as $X(t)=\sum_{i=1}^{k} \operatorname{IMF}_{\mathbf{k}}(\mathbf{t})+\hat{\gamma}_{k}(t)$

Since the EMD method is subject to mode mixing, the ensemble EMD (EEMD) method is proposed to alleviate this problem by adding white noise to the original signal. However, EEMD with high computational cost and the number of decomposed IMFs varies with the added noise. To overcome the aforementioned problem, an improved EEMD method is proposed (Wang et al., 2020b). Let $\boldsymbol{w}^{\boldsymbol{i}}, i=1, \ldots, I$ be white noise with standard deviation $\boldsymbol{\varepsilon}_{\boldsymbol{j}}$. Based on the EMD, the process of cEEMDan can be described as following. Add white noise into the original signal, then the signals being analyzed are $\tilde{\boldsymbol{a}}_{\mathbf{0}}^{\boldsymbol{i}}(\boldsymbol{t})=X(t)+\boldsymbol{\varepsilon}_{\mathbf{0}} \boldsymbol{w}^{\boldsymbol{i}}(\boldsymbol{t}), i=1, \ldots, I$. Using EMD decompose $\tilde{\boldsymbol{a}}_{\mathbf{0}}^{\boldsymbol{i}}$ to obtain its first IMF, denoted as $\widetilde{\mathbf{I M F}}_{1}^{i}$. Then, the first IMF after cEEMDan of $X(t)$ is $\overline{\mathbf{I M F}}_{\mathbf{1}}(\boldsymbol{t})=\frac{1}{I} \sum_{i=\mathbf{1}}^{I} \overline{\mathbf{I M F}}_{\mathbf{1}}^{i}(\boldsymbol{t})$. And the residual after first decomposition is $\tilde{r}_{1}(t)=X(t)-\overline{\mathbf{I M F}}_{\mathbf{1}}(\boldsymbol{t})$. Let $\tilde{r}_{1}$ as the signal need further decomposition, construct the signal by the $\tilde{\boldsymbol{a}}_{1}^{i}(\boldsymbol{t})=\tilde{r}_{1}(t)+\varepsilon_{1} E_{1}\left[\boldsymbol{w}^{i}(t)\right], i=1, \ldots, I$, where $E_{1}(\cdot)$ represents the first IMF obtained by EMD method. The second IMF can be calculated as $\overline{\operatorname{IMF}}_{2}(\boldsymbol{t})=\sum_{i=1}^{I} E_{1}\left[\tilde{\boldsymbol{a}}_{1}^{i}(\boldsymbol{t})\right] / I$. For $k=2, \ldots, K$, calculate the $k$-th residue by $\tilde{r}_{k}(t)=\tilde{r}_{k-1}(t)-\overline{\mathbf{I M F}}_{\boldsymbol{k}}(\boldsymbol{t})$, and decompose $\tilde{\boldsymbol{a}}_{\boldsymbol{k}}^{\boldsymbol{i}}(\boldsymbol{t})=\tilde{r}_{k}(t)+$ $\varepsilon_{k} E_{k}\left[\boldsymbol{w}^{i}(t)\right]$, then $(k+1)$-th IMF can be computed as $\overline{\mathbf{I M F}}_{\boldsymbol{k}+\mathbf{1}}(\boldsymbol{t})=\frac{1}{I} \sum_{i=1}^{I} E_{1}\left[\tilde{\boldsymbol{a}}_{\boldsymbol{k}}^{\boldsymbol{i}}(\boldsymbol{t})\right]$, where $E_{k}(\cdot)$ is the $k$-th IMF obtained by EMD. Repeat the decomposition processes until the residue cannot be further decomposed. After decomposition, the given signal $X(t)$ can be expressed as $X(t)=\sum_{k=1}^{K} \overline{\mathbf{I M F}}_{\boldsymbol{k}}(\boldsymbol{t})+\tilde{r}_{K}(t)$, where $\tilde{r}_{K}(t)$ is the final residue that is no longer feasible to be decomposed. Compared with 
TABLE 2 | List of nomenclature.

\begin{tabular}{|c|c|c|c|}
\hline ADMS & Atmospheric dispersion modelling system & LSTM & Long short-term memory neural network \\
\hline AERMOD & $\begin{array}{l}\text { American meteorological society environmental policy agency regulatory } \\
\text { model }\end{array}$ & $\ell_{2,1}$ RFelm & $\begin{array}{l}\ell_{2,1} \text {-norm and Random fourier mapping-based extreme learning } \\
\text { machine }\end{array}$ \\
\hline $\mathrm{AIC}$ & Akaike information criterion & MA & Moving average model \\
\hline AR & Auto-regressive model & MAE & The mean absolute error \\
\hline ARima & Auto-regressive integrated moving average & MAPE & The mean absolute percentage error \\
\hline BPnn & Back-propagation neural network & MLP & Multi-layer perceptron model \\
\hline CEEMD & Complementary ensemble empirical mode decomposition & MLR & Multiple linear regression \\
\hline cEEMDan & Complete ensemble empirical mode decomposition with adaptive noise & MRMR & Max-relevance and min-redundancy \\
\hline CRTM & Community radiative transfer model & $\mathrm{mSSa}$ & Multi-objective salp swarm algorithm \\
\hline $\mathrm{DM}$ & Diebold-mariano test & ORelm & Outlier-robustness extreme learning machine \\
\hline EEMD & Ensemble empirical Mode decomposition & PM & Particulate matters \\
\hline EMD & Empirical mode decomposition & PRD & The pearl river delta in China \\
\hline Enn & Elman neural network & QD & Quartile deviation \\
\hline ESn & Echo state network & RMSE & The root mean squared error \\
\hline GA & Genetic algorithm & SARima & Seasonal ARima \\
\hline GM & Grey model & SCA & Sine cosine algorithm \\
\hline GZ & Guangzhou & SD & Standard deviation \\
\hline HFs & Fuzzy time series forecasting based on hesitant fuzzy sets & SVR & Support vector regression \\
\hline $\mathrm{HI}$ & Hample Identifier & SZ & Shenzhen \\
\hline HW & Holt-winters & VMD & Variational mode decomposition \\
\hline IEWT & Inverse empirical wavelets transform & VR & Variance ratio \\
\hline IMFs & Intrinsic mode functions & $\begin{array}{l}\text { WRF- } \\
\text { Chem }\end{array}$ & Weather research and forecasting model coupled to chemistry \\
\hline LA & Lichtenberg algorithm & $\mathrm{ZH}$ & Zhuhai \\
\hline
\end{tabular}

cEEMD, cEEMDan has reduced the computational cost (Torres et al., 2011), and Wang et al. (2014) has proved that the computational complexity of EEMD is equivalent to $K \mathcal{O}(T \log T)$, where $T$ is the number of the sample. Therefore, the computational complexity of cEEMDan is less than $K \mathcal{O}(T \log T)$.

\subsection{Individual Forecasting Methods}

In this study, three different types of methods are utilized to predict the concentration of $\mathrm{PM}_{2.5}$. More details are introduced in the following subsections.

\subsubsection{Conventional Statistical Method}

This kind of method is based on statistics, with the advantages of low complexity and fast computational speed, and has a strong model interpretation. One of the most popular and important models is the Auto-regressive Integrated Moving Average (ARima), which has been widely used in time series forecasting (Pai and Lin, 2005; Ariyo et al., 2014; Benvenuto et al., 2020).

For the ARima model, future values are considered as a linear combination of past values and errors, and the mathematical form of the model for predicting is expressed as follows (Pai and Lin, 2005):

$$
\begin{aligned}
X(t)= & \overline{\overline{\boldsymbol{\Psi}}}^{0}+\overline{\overline{\boldsymbol{\Psi}}}^{1} X(t-1)+\overline{\overline{\boldsymbol{\Psi}}}^{2} X(t-2)+\cdots+\overline{\overline{\boldsymbol{\Psi}}}^{p} X(t-p)+\overline{\overline{\boldsymbol{\Psi}}}^{t} \\
& -\overline{\overline{\boldsymbol{\theta}}}^{1} \mathbf{\Upsilon}_{\boldsymbol{t}-\mathbf{1}}-\overline{\overline{\boldsymbol{\theta}}}^{2} \mathbf{\Upsilon}_{\boldsymbol{t}-\mathbf{2}}-\cdots-\overline{\overline{\boldsymbol{\theta}}}^{\boldsymbol{q}} \mathbf{\Upsilon}_{\boldsymbol{t}-\boldsymbol{q}},
\end{aligned}
$$

cwhere $X(t), \ldots, X(t-p)$ are actual values, $\Upsilon_{\boldsymbol{t}}, \ldots, \mathbf{\Upsilon}_{\boldsymbol{t}-\boldsymbol{q}}$ are random errors, $\overline{\mathbf{\Psi}}$ is the trend component, $p$ and $q$ are the order of the auto-regressive model (AR) and moving average model (MA), respectively. For ARima, the complexity is depended on the order of AR $p$ and the order of MA $q$. When the number of sample is $T$, the computational complexity of ARima is $\mathcal{O}\left((T-p) p^{2}+(T-q) q^{2}\right)$ (Gavirangaswamy et al., 2013).

\subsubsection{Fuzzy Computation and Forecasting}

The fuzzy time series forecasting method was first proposed by Song et al. (Song and Chissom, 1993) based on the fuzzy set theory (Zadeh, 1996). It has been continuously developed in recent decades and has been widely applied for forecasting in many fields (Singh, 2007; Cheng et al., 2016; Wang et al., 2021a). As an extension of the fuzzy sets, Torra et al. introduced the concept of hesitant fuzzy sets in 2009 (Torra and Narukawa, 2009). The specific operation steps of HFs are described as follows (Bisht and Kumar, 2016; Cheng et al., 2016; Wang et al., 2021a).

Define the universe of discourse as $U=\left(X_{\min }-\sigma, X_{\max }+\sigma\right)$. Here $X_{\min }$ and $X_{\max }$ are the minimum and maximum of the training set, $\sigma$ is the standard deviation of $X$. Next, using equal and unequal intervals, and triangular membership function to fuzzify the universe of discourse. The length of equal intervals is determined by the distance between the maximum and minimum values in the time series, and the length of unequal intervals is determined by using the cumulative probability distribution approach (Lu et al., 2015; Bisht and Kumar, 2016). Suppose it is divided into $J$ intervals, each interval defined by three parameters, $\underline{\underline{x}}_{l j}$ and $\underline{\underline{x}}_{r j}$ for feet of intervals, and $\overline{\overline{\boldsymbol{x}}}_{m j}$ for the tip of intervals. Two expresses mathematical formula of the triangular membership function (Wang et al., 2021a): 


$$
f(x)= \begin{cases}0 & x<\underline{\underline{x}}_{l j}, \\ \frac{x-\underline{\underline{x}}_{l j}}{\overline{\overline{\boldsymbol{x}}}_{m j}-\underline{\underline{\boldsymbol{x}}}_{l j}} & \underline{\underline{x}}_{l j} \leq x \leq \overline{\overline{\boldsymbol{x}}}_{m j}, \\ \underline{\underline{\boldsymbol{x}}}_{r j}-x & \overline{\overline{\boldsymbol{x}}}_{m j}<x \leq \underline{\underline{\boldsymbol{x}}}_{r j}, \\ \underline{\underline{\boldsymbol{x}}}_{r j}-\overline{\overline{\boldsymbol{x}}}_{m j} & x>\underline{\underline{\boldsymbol{x}}}_{r j} . \\ 0 & \end{cases}
$$

After this step, the membership degrees of $x_{i}$ to equal intervals and the unequal intervals can be obtained, denoted as $\boldsymbol{m} \boldsymbol{d}_{\boldsymbol{e}}$ and $\boldsymbol{m}_{\boldsymbol{u}}$, respectively. Then, compute the weights of equal intervals and unequal intervals using the following formula (Bisht and Kumar, 2016),

$$
\left\{\begin{array}{l}
w_{e}^{j}=\frac{\grave{\boldsymbol{d}}_{e j}}{\grave{\boldsymbol{d}}_{e j}+\grave{\boldsymbol{d}}_{u j}}, \\
w_{u}^{j}=1-w_{e j},
\end{array}\right.
$$

where $d_{e j}$ and $d_{u j}$ are the lengths of $j$-th equal and unequal intervals, $w_{e}^{j}$ and $w_{u}^{j}$ are the weights of $j$-th equal and unequal intervals, respectively. Determine the membership of every element by using aggregate hesitant fuzzy elements, and build a fuzzy set using a novel aggregation operator, which is defined as follows (Wang et al., 2021a):

$$
\begin{aligned}
\boldsymbol{O}\left(x_{1}, x_{2}, \ldots, x_{n}\right) & =1-\left(1-\boldsymbol{m} \boldsymbol{d}_{\boldsymbol{e}}^{i j}\right)^{w_{e}^{j}}\left(1-\boldsymbol{m} \boldsymbol{d}_{\boldsymbol{u}}^{i j}\right)^{w_{u}^{j}}, i=1, \ldots, n ; j \\
& =1, \ldots, J
\end{aligned}
$$

where $\boldsymbol{m} \boldsymbol{d}_{\boldsymbol{e}}^{i j}$ is the membership degree of $x_{i}$ to $j$-th equal interval, so as $\boldsymbol{m} \boldsymbol{d}_{\boldsymbol{u}}^{i j}$ is the membership degree of $x_{i}$ to $j$-th unequal interval, $w_{e}^{j}$ represents the weight of $j$-th equal interval and $w_{u}^{j}$ represents the weight of $j$-th unequal interval. Specifically, $w_{e}^{j} \in[0,1]$, $\sum_{j=1}^{J} w_{e}^{j}=1$.

Following example introduces the specific aggregation process for aggregation:

Let $X=\left\{x_{1}, x_{2}, x_{3}\right\}$ be a reference set. $\mathcal{H}=$ $\left\{\left\langle x_{1},\{0.2,0.4\}\right\rangle,\left\langle x_{2},\{0.5,0.25\}\right\rangle,\left\langle x_{3},\{0.3,0.4\}\right\rangle\right\}$ is a hesitant fuzzy set on $\mathrm{X}$, and taking $\mathrm{w}=(0.4,0.6)$. Applying the aggregation method motioned above, the fuzzy elements can be obtained as follows:

$$
\begin{aligned}
& \boldsymbol{h}\left(\boldsymbol{x}_{1}\right)=1-(1-0.2)^{\frac{2}{5}}(1-0.4)^{\frac{3}{5}} \approx 0.33 \\
& \boldsymbol{h}\left(\boldsymbol{x}_{2}\right)=1-(1-0.5)^{\frac{2}{5}}(1-0.25)^{\frac{3}{5}} \approx 0.41 \\
& \boldsymbol{h}\left(\boldsymbol{x}_{3}\right)=1-(1-0.3)^{\frac{2}{5}}(1-0.4)^{\frac{3}{5}} \approx 0.64
\end{aligned}
$$

Therefore, the fuzzy set $\mathcal{A}$ is established as $\mathcal{A}=\left\{\left\langle x_{1}, 0.33\right\rangle,\left\langle x_{2}, 0.41\right\rangle,\left\langle x_{3}, 0.64\right\rangle\right\}$.

After determining the membership of every element, establish fuzzy logical relationships and fuzzy logical relationship groups. The fuzzy logical relationships are established by the rule: If $\boldsymbol{A}_{\boldsymbol{i}}$ and $\boldsymbol{A}_{\boldsymbol{i}+\boldsymbol{1}}$ are the fuzzy values at time $t$ and $t+1$ respectively, the fuzzy logical relation is denoted as $\boldsymbol{A}_{\boldsymbol{i}} \rightarrow \boldsymbol{A}_{\boldsymbol{i}+\boldsymbol{1}}$. Here, $\boldsymbol{A}_{\boldsymbol{i}}$ is called the current state and $A_{i+1}$ is the next state. Then, the same left-hand side of the fuzzy
TABLE 3 | The process of the HFs.

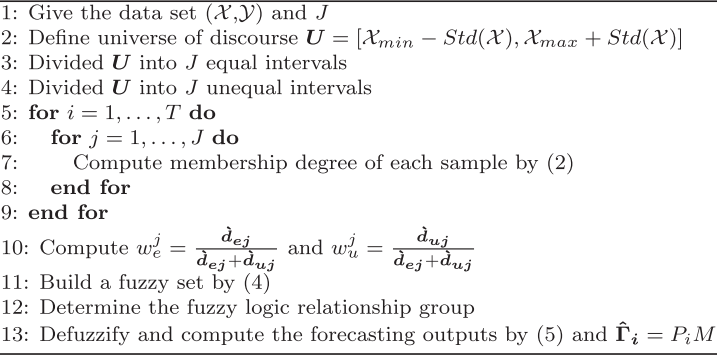

logical relationships is classified to form several fuzzy logical relationship groups. The main idea of forecasting is to infer the next state based on the current state. Based on the fuzzy logical relationship groups, a matrix $P_{m \times m}$ can be generated, each element in $P$ represents the frequency of $\boldsymbol{A}_{\boldsymbol{i}} \rightarrow \boldsymbol{A}_{\boldsymbol{i}+\boldsymbol{1}}$ that with the same fuzzy logical relationship. According to the max-min composition operations on fuzzy logical relationship, the fuzzy output can be obtained and defuzzify by $\hat{\boldsymbol{\Gamma}}_{\mathbf{i}}=P_{i} M$, here $M$ is the combined midpoint of the triangular membership functions for equal and unequal intervals respectively, calculated as follows (Bisht and Kumar, 2016):

$$
M=\frac{M_{e} w_{e}+M_{u} w_{u}}{w_{e}+w_{u}}
$$

where $M_{e}$ and $M_{u}$ is the mid points of the equal and unequal intervals. As the introduction above, the computational complexity of HFs is $\mathcal{O}(J n), J$ is the number of the interval and $n$ represents the number of sample.

Summarizing all this activity, Table $\mathbf{3}$ is given to show the implementation of the HFs.

\subsubsection{Machine Learning Technique}

The methods based on machine learning have strong learning ability and can handle the non-linear components in the time series, so they have been widely used in some fields (Gündüz et al., 2019; Henrique et al., 2019; Volk et al., 2020; Wang et al., 2021b). In this study, three different networks were selected to analyze the series, since the features of the series are uncertain.

\section{$(\mathcal{A})$ Back-Propagation Neural Network}

Back-propagation neural network (BPnn) is a three-layer feed-forward network with an input layer, a hidden layer, and an output layer. Each layer takes inputs only from the previous layer and sends the outputs only to the next layer. Define the input vector as $\mathcal{X}=\left\{\boldsymbol{X}_{1}, \boldsymbol{X}_{2}, \ldots, \boldsymbol{X}_{N}\right\}$, and the output vector as $\mathcal{Y}=\left\{\boldsymbol{Y}_{1}, \boldsymbol{Y}_{2}, \ldots, \boldsymbol{Y}_{N}\right\}$. Assume the input layer has $I$ neurons, the hidden layer has $H$ neurons, and the output layer has one neuron, the network can be constructed as Figure $\mathbf{1 B}$, and the training processes are described as follows. 


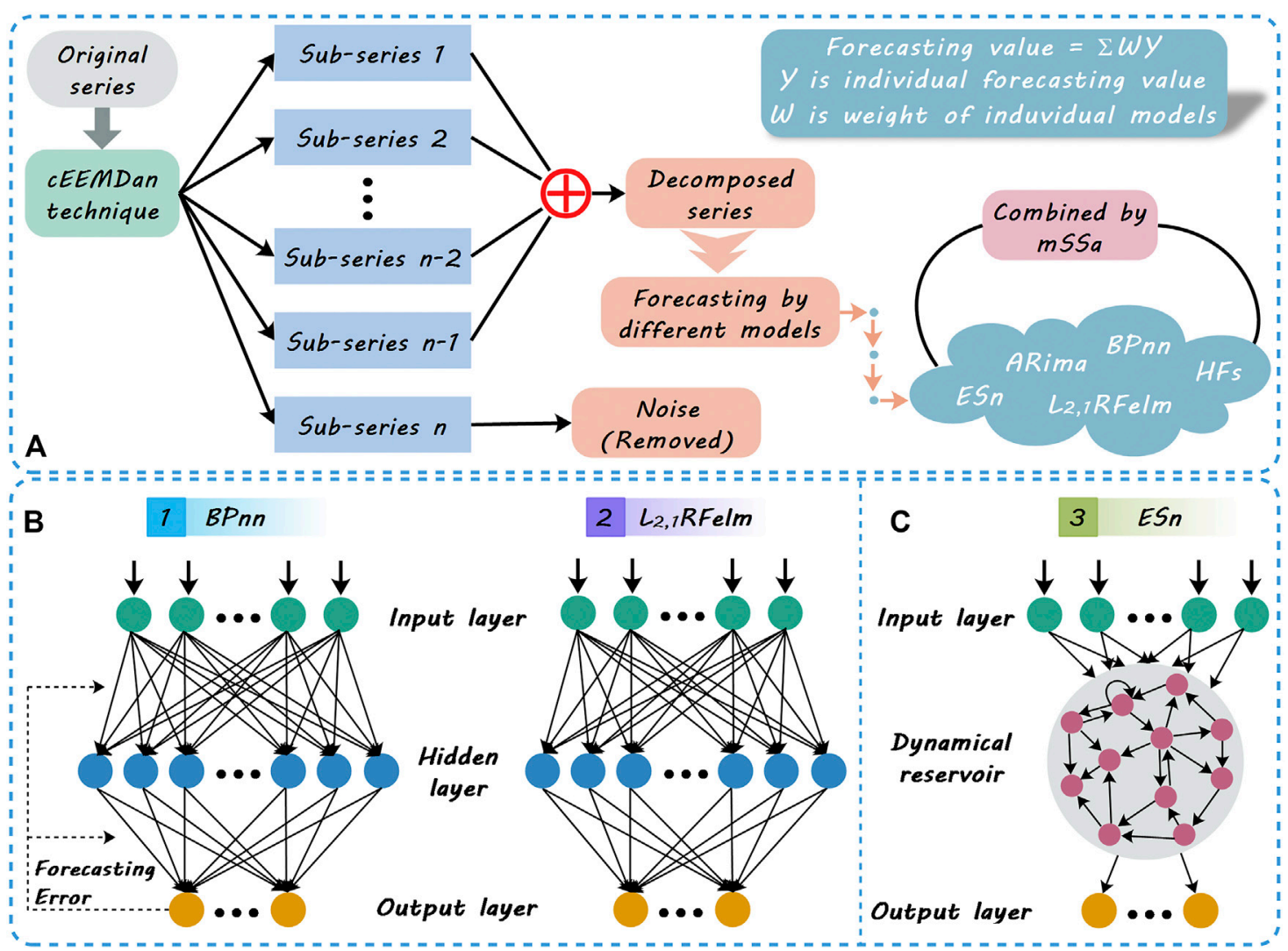

FIGURE 1 | (A) is the flowchart of the proposed combined system; (B) and (C) are the structures of the neural networks used in this paper, where BPnn and $\ell_{2,1}$ RFelm have the same structure and different solution processes.

Calculate outputs of all neurons in hidden layer (HechtNielsen, 1992; Wang Y. et al., 2021):

$$
\left\{\begin{array}{l}
\boldsymbol{h}_{\boldsymbol{h}}^{\boldsymbol{i}}=\sum_{i=1}^{I} w_{i h} x_{n}+\overline{\boldsymbol{\mho}}_{h}, h=1,2, \ldots, H, \\
\boldsymbol{h}_{\boldsymbol{h}}^{\boldsymbol{o}}=f\left(\boldsymbol{h}_{\boldsymbol{h}}^{i}\right), h=1,2, \ldots, H,
\end{array}\right.
$$

Where, $\boldsymbol{h}_{\boldsymbol{h}}^{i}$ is the activation value of the $h$-th node of hidden layer, $\boldsymbol{h}_{h}^{o}$ represents the output value of $h$-th hidden neuron, $w_{i h}$ denotes the connection weight between $i$-th input neuron and $h$-th hidden neuron, $\overline{\boldsymbol{\sigma}}_{h}$ represents the bias of $h$-th hidden neuron, and $f(\cdot)$ is the activation function. Then, determine the output of the network as $\boldsymbol{O}^{\boldsymbol{o}}=g\left(\sum_{h=1}^{H} w_{h} \boldsymbol{h}_{\boldsymbol{h}}^{\boldsymbol{o}}+\overline{\overline{\boldsymbol{O}}}\right)$, where $\boldsymbol{O}^{\boldsymbol{o}}$ is the output value of output neuron, $w_{h}$ is the weight between $h$-th hidden neuron and output neuron, $\overline{\bar{\sigma}}$ represents the bias of output neuron, and $g(\cdot)$ is the activation function. Obtain the minimum global error by the "error feedback" training mechanism. The global error is $\mathcal{E}=\sum_{n=1}^{N}\left(\boldsymbol{O}_{n}^{\boldsymbol{o}}-\boldsymbol{Y}_{n}\right)^{2} / 2, \boldsymbol{O}_{n}^{\boldsymbol{o}}$ represents $n$-th output of network. For more details, please refer to (Hecht-Nielsen, 1992). For each iteration, the computational complexity of both the forward propagation process and the backward propagation process is $\mathcal{O}(N(H+1)(F+1)+N(H+1)(O+1))$, where $F$ is the dimension of the input set and $O$ represents the dimension of the output set. In this study, $F=4, O=1$, so the computational complexity of the algorithm is $T_{b p n n} \mathcal{O}(N H)$, here $T_{b p n n}$ is the number of iterations.

\section{(B) $\mathfrak{\ell}_{2,1}$-Norm and Random Fourier Mapping-Based Extreme} Learning Machine

$\ell_{2,1}$ RFelm is an improved feed-forward neural network with a single hidden layer, which was proposed by Zhou et al. (2016). In this method, Random Fourier Mapping is used to improve the extendibility of the network by approximating the activation function in ELM. And $\ell_{2,1}$-norm is used to make the hidden layer more compact and discriminative by cutting irrelevant neurons.

To predict the $\mathrm{PM}_{2.5}$ concentration of the day, the $\mathrm{PM}_{2.5}$ concentrations of the past 4 days are used. So, the original concentration series $X=\left\{x_{1}, x_{2}, \ldots, x_{T}\right\}$ is reconstructed as follows:

$$
\begin{gathered}
\mathcal{X}=\left[\boldsymbol{X}_{\mathbf{1}}, \boldsymbol{X}_{2}, \ldots, \boldsymbol{X}_{\boldsymbol{N}}\right]=\left[\begin{array}{llll}
x_{1} & x_{2} & \cdots & x_{T-4} \\
x_{2} & x_{3} & \cdots & x_{T-3} \\
x_{3} & x_{4} & \cdots & x_{T-2} \\
x_{4} & x_{5} & \cdots & x_{T-1}
\end{array}\right], \\
\mathcal{Y}=\left[\boldsymbol{Y}_{\mathbf{1}}, \boldsymbol{Y}_{2}, \ldots, \boldsymbol{Y}_{\boldsymbol{N}}\right]=\left[x_{5}, x_{6}, \ldots, x_{T-1}, x_{T}\right] .
\end{gathered}
$$

Then, the main processes of this method can be introduced as follows:

Randomly initialize the connection weights $\mathcal{W}$ between the input layer and hidden layer and the bias $\mathcal{B}$ of the hidden layer, 
assume the hidden layer has $H$ neurons, these two matrix are represented as follows:

$$
\begin{gathered}
\mathcal{W}=\left[W_{1}, \ldots, W_{h}, \ldots, W_{H}\right]^{\mathrm{T}}=\left[\begin{array}{cccc}
w_{11} & w_{12} & w_{13} & w_{14} \\
w_{21} & w_{22} & w_{23} & w_{24} \\
\vdots & \vdots & \vdots & \vdots \\
w_{H 1} & w_{H 2} & w_{H 3} & w_{H 4}
\end{array}\right], \\
\mathcal{B}=\left[b_{1}, \ldots, b_{h}, \ldots, b_{H}\right], \quad h=1,2, \ldots, H,
\end{gathered}
$$

here $w_{H 1}$ represents the weight between the first input neuron and $H$-th hidden neuron, $b_{h}$ represents the bias of $h$-th hidden neuron. Then the output matrix of the hidden layer is

$$
\mathcal{H}=\left[\begin{array}{cccc}
g\left(W_{1} \boldsymbol{X}_{1}+b_{1}\right) & g\left(W_{1} \boldsymbol{X}_{2}+b_{1}\right) & \cdots & g\left(W_{1} \boldsymbol{X}_{N}+b_{1}\right) \\
g\left(W_{2} \boldsymbol{X}_{1}+b_{2}\right) & g\left(W_{2} \boldsymbol{X}_{2}+b_{2}\right) & \cdots & g\left(W_{2} \boldsymbol{X}_{N}+b_{2}\right) \\
\vdots & \vdots & \ddots & \vdots \\
g\left(W_{H} \boldsymbol{X}_{1}+b_{H}\right) & g\left(W_{H} \boldsymbol{X}_{2}+b_{H}\right) & \cdots & g\left(W_{H} \boldsymbol{X}_{N}+b_{H}\right)
\end{array}\right] .
$$

In this method, the Random Fourier Mapping $g(\cdot)$ is used to approximate the kernel function, so $\mathcal{W} \mathcal{X}+\mathcal{B}$ can be mapped into a Random Fourier feature space. The specific mapping is defined as below (Rahimi and Recht, 2007):

$g(x)=\frac{1}{\sqrt{N}}\left[\cos \left(w_{1}^{\mathrm{T}} x\right), \ldots, \cos \left(w_{N}^{\mathrm{T}} x\right), \sin \left(w_{1}^{\mathrm{T}} x\right), \ldots, \sin \left(w_{N}^{\mathrm{T}} x\right)\right]^{\mathrm{T}}$

Then, calculate the output of the network and solve parameter. Let the connection weight between the hidden layer and the output layer is $\boldsymbol{\beta}=\left[\beta_{1}, \beta_{2}, \ldots, \beta_{H}\right]^{\mathrm{T}}$, then the output function of this network is $\sum_{h=1}^{H} \beta_{h} g\left(W_{h} \boldsymbol{X}_{\boldsymbol{n}}+\overline{\boldsymbol{\sigma}}_{h}\right)=O_{i}, n=1,2, \ldots, N$. In $\ell_{2,1}$ RFelm, the only parameter need to solve is $\beta$. Based on the given data and the initial parameters, the objective function of this network is

$$
\begin{gathered}
\underset{\boldsymbol{\beta}, \varepsilon}{\operatorname{Min}} \frac{1}{2}\|\boldsymbol{\beta}\|_{2,1}+\frac{1}{2} \tilde{\mathrm{C}} \sum_{i=1}^{N}\left\|\Upsilon_{i}\right\|^{2}, \\
\text { s.t. } g\left(\boldsymbol{X}_{i}\right) \boldsymbol{\beta}=y_{i}-\Upsilon_{i}, i=1,2, \ldots, \mathrm{N},
\end{gathered}
$$

chere $\varepsilon$ represents the training error, $\tilde{C}$ is the penalty coefficient, and $\left\|\boldsymbol{\beta}^{\mathrm{T}}\right\|_{2,1}$ is $\ell_{2,1}$-norm of $\boldsymbol{\beta},\|\boldsymbol{\beta}\|_{2,1}=\sqrt{\sum_{h=1}^{H} \beta_{h}^{2}}$. Finally, $\beta$ can be obtained $\hat{\boldsymbol{\beta}}=\left(\mathbf{D} / \tilde{\mathrm{C}}+\mathcal{H}^{\mathrm{T}} \mathcal{H}\right)^{-1} \mathcal{H}^{\mathrm{T}} \mathcal{Y}^{\mathrm{T}}$, where $\mathbf{D}$ is a diagonal matrix with $\mathbf{D}_{h h}=1 /\left(2\|\boldsymbol{\beta}\|_{2}\right)$, and at the beginning of the iterative, $\mathbf{D}$ is an identity matrix. For more details of solve process, please refer to (Zhou et al., 2016). In this study, the computational complexity of $\ell_{2,1}$ RFelm is mainly contributed by the process of computing $\left(\mathrm{D} / \tilde{\mathrm{C}}+\mathcal{H}^{\mathrm{T}} \mathcal{H}\right)^{-1}$. Thus the computational complexity of $\ell_{2,1}$ RFelm is $T_{r f e l m} \mathcal{O}\left(H^{3}\right), T_{r f e l m}$ is the number of the iterations.

\section{(C) Echo State Network}

Echo state network (ESn) is an improved recurrent neural network and was proposed in 2004 (Jaeger and Haas, 2004). Without output feedback connections, an ESn consists of an input layer with $I$ neurons, $L$ internal neurons possessing internal states, and one output neuron. The structure of ESn is shown in Figure 1C. Given a training set $[\mathcal{X}, \mathcal{Y}]$ the main steps of ESn are as follows (Qiao et al., 2016; Wang et al., 2019). Randomly generate a reservoir weight matrix $W$ with the predefined sparsity and size. In order for the reservoir to have echo-state property, the singular values of reservoir weight matrix of the reservoir must be scaled to within 1 , so scaled $W$ as $\tilde{\mathbf{W}}=(\boldsymbol{\alpha} / \Psi) \mathbf{W}$, here $0<\boldsymbol{\alpha}<1$ and $\boldsymbol{\Psi}$ is the spectral radius of $\boldsymbol{W}$. Next, randomly generate the weight matrix between input layer and reservoir, denoted as $W^{i n}$. And initialize the reservoir states $\mathfrak{E}(\mathbf{0})$. Calculate the state of reservoir by using dynamic equation, $\mathfrak{E}(\mathbf{n}+\mathbf{1})=\mathcal{F}(\tilde{\boldsymbol{W}}$ $\left.\mathfrak{E}(\mathbf{n})+\boldsymbol{W}^{i n} \boldsymbol{X}_{n+1}\right)$, here $\mathfrak{E}(\mathbf{n})$ and $\mathfrak{E}(\mathbf{n}+\mathbf{1})$ are reservoir states, $\mathcal{F}(\cdot)$ is activation function, $\boldsymbol{X}_{\boldsymbol{n}+\boldsymbol{1}}$ represent $(n+1)$-th sample input. Finally, calculate the network output $\hat{y}_{n+1}=\mathcal{G}\left(\boldsymbol{W}^{\text {out }} \mathfrak{E}(\mathbf{n}+\mathbf{1})\right)$, where $\boldsymbol{W}^{\text {out }}$ represents weight matrix between reservoir and output layer, $\mathcal{G}(\cdot)$ is activation function. The only trainable part of the ESn is the output weight matrix $W^{\text {out }}$, and can be commonly obtained as $\boldsymbol{W}^{\text {out }}=\left(\mathcal{X}^{\mathrm{T}} \mathcal{X}\right)^{-1} \mathcal{X}^{\mathrm{T}} \mathcal{Y}$. As shown above, the computational complexity of ESn is largely proportional to the state updating process, the complexity of this process is equal to $\mathcal{O}(L T)$, where $T$ is the number of sample.

\subsection{Optimization of Combination Weights}

Mirjalili et al. proposed a novel swarm intelligence optimization algorithm in 2017, which was inspired by the behavior of salps looking for food (Mirjalili et al., 2017a). Their study has shown that this method can approximate the Pareto optimal solution with high convergence and coverage. It has merits among the current optimization algorithms and is worth applying to different problems (Mirjalili et al., 2017a). Therefore, this method (mSSa) is used to find the optimal combined weight of different forecasting models in this study. More details are introduced as follows.

\subsubsection{Multi-Objective Optimization}

Multi-objective optimization is concerned with mathematical optimization problems involving more than one objective function to be optimized simultaneously (Haimes et al., 2011). The multi-objective optimization problem can represent as follows:

$$
\left\{\begin{array}{l}
\text { Minimize }\left[\mathbf{O b f}_{1}(x), \mathbf{O b f}_{2}(x), \ldots, \mathbf{O b f}_{o}(x)\right] \\
\text { subject to } x \in \mathcal{S}
\end{array}\right.
$$

where $\mathcal{S}$ is the feasible search space, $o$ is the number of objective function, and $\mathbf{O b f}_{i}$ is $i$-th objective function.

The purpose of multi-objective optimization is to find the set of acceptable solutions (Ngatchou et al., 2005). Hence, the definitions related to the Pareto-optimal solutions are introduced.

Definition 1. Pareto domination Given two vectors $\overrightarrow{\mathbf{X}}=$ $\left(x_{1}, x_{2}, \ldots, x_{n}\right)$ and $\overrightarrow{\mathbf{Y}}=\left(y_{1}, y_{2}, \ldots, y_{n}\right)$, vector $\overrightarrow{\mathbf{Y}}$ dominates $\overrightarrow{\mathbf{X}}$ or called vector $\overrightarrow{\mathbf{X}}$ is dominated by vector $\overrightarrow{\mathbf{Y}}$ denoted as $\overrightarrow{\mathbf{Y}} \prec \overrightarrow{\mathbf{X}}$ if and only if $\forall i \in[1, o],\left[\mathbf{O b f}_{i}(\overrightarrow{\mathbf{Y}}) \leq \mathbf{O b f}_{i}(\overrightarrow{\mathbf{X}})\right] \wedge$ $\exists i \in[1, o],\left[\mathbf{O b f}_{i}(\overrightarrow{\mathbf{Y}})<\mathbf{O b f}_{i}(\overrightarrow{\mathbf{X}})\right]$, where $\mathbf{O b f}_{\mathrm{i}}(\cdot)$ represents i-th objective function.

Definition 2. Pareto optimal set A set including all the nondominated solutions is called Pareto optimal set. The mathematical description is $\mathbf{P}_{s}:=\{x, z \in \overrightarrow{\mathbf{X}} \mid \nexists z \prec x\}$. 
TABLE 4 | Descriptive statistics of data sets.

\begin{tabular}{|c|c|c|c|c|c|c|c|c|c|}
\hline \multirow[t]{2}{*}{ Study areas } & \multirow{2}{*}{$\begin{array}{c}\text { Data sets (number of } \\
\text { obs) }\end{array}$} & \multicolumn{3}{|c|}{ Central tendency } & \multicolumn{3}{|c|}{ Variability } & \multicolumn{2}{|c|}{ Distribution } \\
\hline & & Mean & Median & Mode & SD & Range & QD & Kurt & Skew \\
\hline \multirow[t]{3}{*}{$\mathrm{GZ}$} & All $(3,600)$ & 16.0327 & 12.7419 & 2.1746 & 11.2666 & 60.5663 & 11.5382 & 5.3415 & 1.5700 \\
\hline & Training $(2,522)$ & 14.1506 & 10.1960 & 2.1746 & 11.5619 & 60.5663 & 9.1079 & 7.1258 & 2.0779 \\
\hline & Test $(1,078)$ & 20.4358 & 17.8701 & 5.9780 & 9.1420 & 42.0838 & 13.3895 & 2.7505 & 0.7581 \\
\hline \multirow[t]{3}{*}{$\mathrm{SZ}$} & All $(3,600)$ & 12.5621 & 8.5605 & 1.9515 & 11.1879 & 70.7185 & 10.6374 & 7.1971 & 1.9834 \\
\hline & Training $(2,522)$ & 9.9424 & 6.7614 & 1.9515 & 10.2282 & 70.7185 & 6.0688 & 12.9819 & 3.0015 \\
\hline & Test $(1,078)$ & 18.6908 & 16.4895 & 2.8867 & 10.9422 & 53.1308 & 13.5744 & 3.6718 & 0.9919 \\
\hline \multirow[t]{3}{*}{$\mathrm{ZH}$} & All $(3,600)$ & 13.8872 & 10.5852 & 1.8574 & 72.2932 & 70.4359 & 10.1938 & 6.7821 & 1.8325 \\
\hline & Training $(2,522)$ & 11.6366 & 8.8954 & 1.8574 & 72.2932 & 70.4359 & 6.3972 & 10.6458 & 2.5882 \\
\hline & Test $(1,078)$ & 19.1524 & 17.0813 & 4.3352 & 62.0781 & 57.7429 & 11.9228 & 4.7049 & 1.1551 \\
\hline
\end{tabular}

Note SD: standard deviation; QD: quartile deviation; Kurt. kurtosis; Skew. skewness.

\begin{tabular}{|c|c|c|}
\hline Method & Meaning & Value \\
\hline \multirow[t]{3}{*}{ cEEMDan } & Noise standard deviation & 0.5 \\
\hline & Number of realizations & 200 \\
\hline & Maximum number of sifting iterations allowed & 10 \\
\hline \multirow[t]{3}{*}{ ARima } & The lag order & 10 (GZ), 8 (SZ), 8 (ZH) \\
\hline & The degree of differencing & 1 (GZ), 1 (SZ), 1 (ZH) \\
\hline & The order of the moving average & 7 (GZ), $10(\mathrm{SZ}), 10(\mathrm{ZH})$ \\
\hline HTS & Number of interval & 23 (GZ), 24 (SZ), $23(\mathrm{ZH})$ \\
\hline \multirow[t]{6}{*}{ BPnn } & Maximum number of iteration times & 100 \\
\hline & Learning rate & 0.1 \\
\hline & Training accuracy goal & 0.00001 \\
\hline & Neuron number of input layer & 4 \\
\hline & Neuron number of hidden layer & 9 \\
\hline & Neuron number of output layer & 1 \\
\hline \multirow[t]{3}{*}{$\ell_{2,1}$ RFelm } & Penalty coefficient & 5 \\
\hline & Maximum iterations & 50 \\
\hline & Number of neurons in hidden layer & 15 \\
\hline \multirow[t]{5}{*}{ ESn } & Reservoir dimension & 20 \\
\hline & Spectral radius & 0.2 \\
\hline & Leaking rate & 0.5 \\
\hline & Connectivity & 0.2 \\
\hline & Readout regularization & 0.05 \\
\hline \multirow[t]{4}{*}{$\mathrm{mSSa}$} & Size of archive & 100 \\
\hline & Size of population & 30 \\
\hline & Maximum iterations & 50 \\
\hline & Individual value range & {$[-5,5]$} \\
\hline
\end{tabular}

\subsubsection{Process of Multi-Objective Salp Swarm Algorithm}

The individuals in a salp chain are divided into two groups: the front of the chain is the leader, the others are followers. Assume $\boldsymbol{O}$ indicates the dimension of search space, $\boldsymbol{N}$ denotes the number of salp chains, then the location of all the salps can be defined as a matrix:

$$
\mathcal{P}_{t}=\left[\begin{array}{cccc}
p_{1}^{1}(t) & p_{2}^{1}(t) & \ldots & p_{O}^{1}(t) \\
p_{1}^{2}(t) & p_{2}^{2}(t) & \ldots & p_{O}^{2}(t) \\
\vdots & \vdots & \ldots & \vdots \\
p_{1}^{N}(t) & p_{2}^{N}(t) & \ldots & p_{O}^{N}(t)
\end{array}\right]
$$

here $t$ represents $t$-th iteration. The position of each salp is a candidate solution. Next, calculate fitness of each salp chain Fit $\left[\vec{p}^{j}(t)\right]=\left\{o b f_{1}\left[\vec{p}^{j}(t)\right], \quad o b f_{2}\left[\vec{p}^{j}(t)\right], \ldots, o b f_{O}\left[\vec{p}^{j}(t)\right]\right\}$ $j=1,2, \ldots, N$, where $\operatorname{Fit}\left[\vec{p}^{j}(t)\right]$ represents the fitness of $j$-th salp chain at $t$-th iteration, $o b f_{o}\left[\vec{p}^{j}(t)\right]$ is the value of $o$-th objective function of $j$-th salp chain at $t$-th iteration. Then, determine the non-dominated salp chains according to Definition 1, and update the archive (Pareto optimal set, Definition 2). Select a salp chain as a food source from the archive, denoted as $\mathbf{F}$. After that, leaders $p_{1}$ guides the salp swarm toward the food source in an $\boldsymbol{O}$-dimensional search space. The positions of the leaders are updated as follows (Mirjalili et al., 2017a):

$$
p_{1}^{i}(t+1)= \begin{cases}\mathbf{F}_{i}(t)+\boldsymbol{\tau}_{1}\left[\left(\overline{\overline{p_{1}}}-\underline{\underline{p}_{1}}\right) \boldsymbol{\tau}_{2}+\underline{\underline{p}}_{1}\right], & \boldsymbol{\tau}_{3} \geq 0, \\ \mathbf{F}_{i}(t)-\boldsymbol{\tau}_{1}\left[\left(\overline{\overline{p_{1}}}-\underline{\underline{\underline{p}}}\right) \boldsymbol{\tau}_{2}+\underline{\underline{\underline{p}}} 1\right], & \boldsymbol{\tau}_{3}<0,\end{cases}
$$


TABLE 6 | Forecasting results of individual models and combined systems based on the original data and decomposed data.

\begin{tabular}{|c|c|c|c|c|c|c|c|c|c|}
\hline & \multicolumn{3}{|c|}{ GZ } & \multicolumn{3}{|c|}{ SZ } & \multicolumn{3}{|c|}{$\mathbf{Z H}$} \\
\hline & MAE & RMSE & MAPE & MAE & RMSE & MAPE & MAE & RMSE & MAPE \\
\hline \multicolumn{10}{|c|}{ (a1) Individual models without cEEMDan } \\
\hline ARima & 1.3628 & 1.8714 & 7.4792 & 1.8384 & 2.6862 & 12.0589 & 1.6974 & 2.3692 & 10.2120 \\
\hline BPnn & 2.1184 & 2.9313 & 11.3664 & 3.0927 & 4.4142 & 19.3875 & 2.6765 & 3.8144 & 15.7448 \\
\hline$\ell_{2,1}$ RFelm & 2.1446 & 2.9753 & 11.4354 & 2.9969 & 4.2871 & 18.9209 & 2.6160 & 3.7088 & 15.4394 \\
\hline ESn & 2.1333 & 2.9944 & 11.4066 & 2.9570 & 4.1990 & 18.7598 & 2.6277 & 3.7268 & 15.4739 \\
\hline HFs & 1.7617 & 2.2905 & 9.6849 & 1.6525 & 2.0928 & 10.7961 & 2.1028 & 3.0032 & 11.4203 \\
\hline \multicolumn{10}{|c|}{ (a2) Individual models combined with cEEMDan } \\
\hline C-ARima & 0.4834 & 0.6495 & 2.6661 & 0.6196 & 0.9590 & 4.0504 & 0.5907 & 0.8305 & 3.5916 \\
\hline C-BPnn & 1.0783 & 1.4473 & 5.8968 & 1.5525 & 2.1486 & 9.9900 & 1.4011 & 1.8842 & 8.3322 \\
\hline C- $\ell_{2,1}$ RFelm & 0.9548 & 1.3033 & 5.1095 & 1.3786 & 1.8823 & 8.8777 & 1.2266 & 1.6663 & 7.1065 \\
\hline C-ESn & 1.0301 & 1.4782 & 5.6234 & 1.3923 & 1.9313 & 9.2527 & 1.2675 & 1.8130 & 7.4409 \\
\hline \multicolumn{10}{|c|}{ (b1) Combined system without ceemdan } \\
\hline FIX & 1.4641 & 2.0242 & 6.8942 & 1.8370 & 2.3831 & 8.2947 & 1.8483 & 2.5445 & 8.3929 \\
\hline MAX & 14.7246 & 15.9534 & 63.3391 & 16.5771 & 17.7847 & 71.4600 & 18.1277 & 19.4904 & 80.8421 \\
\hline MIN & 1.5737 & 2.2184 & 7.3353 & 1.8109 & 2.3946 & 8.2507 & 1.7003 & 2.3171 & 7.9920 \\
\hline MIX & 10.6795 & 11.6622 & 45.6769 & 2.7319 & 3.5157 & 11.5748 & 6.3011 & 7.1793 & 27.4367 \\
\hline MRMR & 1.4599 & 2.0244 & 6.8277 & 1.7541 & 2.2987 & 7.9835 & 1.8007 & 2.4644 & 8.3023 \\
\hline ReliefF & 1.2977 & 1.7443 & 6.4876 & 1.4309 & 1.8380 & 6.3749 & 1.6705 & 2.3664 & 7.3116 \\
\hline LA & 1.3942 & 2.3467 & 6.8619 & 1.6659 & 2.0776 & 7.9034 & 1.4789 & 2.2477 & 6.3263 \\
\hline $\mathrm{mSSa}$ & 1.1232 & 1.5157 & 5.8992 & 1.2227 & 1.5390 & 6.4305 & 1.1772 & 1.6712 & 5.2612 \\
\hline \multicolumn{10}{|c|}{ (b2) Combined system includes cEEMDan } \\
\hline C-FIX & 0.7764 & 1.0859 & 3.7301 & 0.9307 & 1.1954 & 4.2834 & 0.9690 & 1.3614 & 4.4141 \\
\hline C-MAX & 3.3711 & 3.7705 & 14.2808 & 9.8564 & 10.5824 & 42.5217 & 11.9701 & 12.8744 & 53.4489 \\
\hline C-MIN & 0.7383 & 0.9943 & 3.4696 & 0.7709 & 1.0495 & 3.6179 & 1.2171 & 1.6056 & 5.6501 \\
\hline C-MIX & 16.0585 & 17.3482 & 69.3189 & 11.8266 & 12.6870 & 51.0301 & 13.5027 & 14.5101 & 60.3564 \\
\hline C-MRMR & 0.6598 & 0.9300 & 3.1344 & 0.7951 & 1.0481 & 3.6887 & 0.8203 & 1.1252 & 3.7768 \\
\hline C-ReliefF & 0.7723 & 1.0531 & 3.8831 & 0.9448 & 1.1974 & 4.1829 & 0.8833 & 1.2485 & 3.9945 \\
\hline C-LA & 0.5027 & 0.6874 & 2.4168 & 0.5837 & 0.8361 & 2.8366 & 0.5760 & 0.7900 & 2.7692 \\
\hline C-mSSa & 0.4776 & 0.6526 & 2.3576 & 0.5670 & 0.8101 & 2.7879 & 0.5642 & 0.7750 & 2.7404 \\
\hline
\end{tabular}

"C-" represents the forecasting models combined with cEEMDan.

The bold numbers indicate the optimal value of the indicators.

where $p_{1}^{i}(t+1)$ is the position of leader in the $i$-th chain at $(t+$ 1)-th iteration, $\mathbf{F}_{i}(t)$ represents the food source position in the $i$-th dimension at $t$-th iteration. $\underline{p}_{1}$ and $\overline{\overline{p_{1}}}$ are the lower bound and the upper bound of $p_{1}$.

In Eq. 14, $\tau_{1}$ is a parameter that controls the balance of exploration and exploitation, $\boldsymbol{\tau}_{2}$ is a random number in $(0,1)$ that determines the distance to move, and $\tau_{3}$ is also a random number in $(0,1)$ that determines the direction of movement. The coefficient $\boldsymbol{\tau}_{1}$ is defined as $\boldsymbol{\tau}_{1}=2 e^{-\left(4 t / T_{m S s a}\right)^{2}}$, where $t$ is the number of the current iteration and $\boldsymbol{T}_{\boldsymbol{m} S \boldsymbol{S} \boldsymbol{a}}$ represents the number of maximum iteration. Whereafter, the positions of the followers are mathematically updated as $p_{j}^{i}(t+1)=\frac{1}{2}\left[p_{j}^{i}(t)+p_{j}^{i-1}(t)\right], \forall 2 \leq j, i=1,2, \ldots, N$. Finally, repeat the processes of calculating fitness, updating the archive, selecting food source and updating the salps location until satisfied with the end condition.

If the archive is not full, the non-dominated solutions are saved to the archive after comparison according to Definition 1, otherwise, before storage deletes some solutions (Mirjalili et al., 2017a). According to the principle of improving the distributivity of solutions in the archive, use the Roulette Wheel mechanism to remove the densest solutions. The probability of the solution being removed can be calculated as $\mathbf{P}_{r}=N_{l} / \mathcal{c}$, where $N_{l}$ is the number of $l$-th solution in the archive, and $c$ is a constant greater than 1 (Mirjalili et al., 2017b).

According to the introduction of $\mathrm{mSSa}$, the computational complexity of this method is $\mathcal{O}\left(O \times N+\operatorname{cof} \times N+2 N^{2}\right)$ at one iteration, where cof is the computational complexity of the objective function. In this study, the Mean Square Error and the Standard deviation of the error are set as objective function. The complexity of the first objective functions is $\mathcal{O}\left(T^{2}\right)$ and the second objective function is $\mathcal{O}(T)$. Therefore, the complexity of one iteration of mSSa is $\mathcal{O}\left(O \times N+\left(T^{2}+T\right) \times N+2 N^{2}\right)$, here $T$ is the number of samples.

\subsection{The Proposed Combined Forecasting System}

Using the aforementioned methods and strategy, a novel combined pollutant concentration forecasting system based on the data decomposition strategy, several individual forecasting models, and a multi-objective optimization algorithm is designed.

Assume there are $M$ models to predict the pollutant concentration, the forecasting results are denoted as 


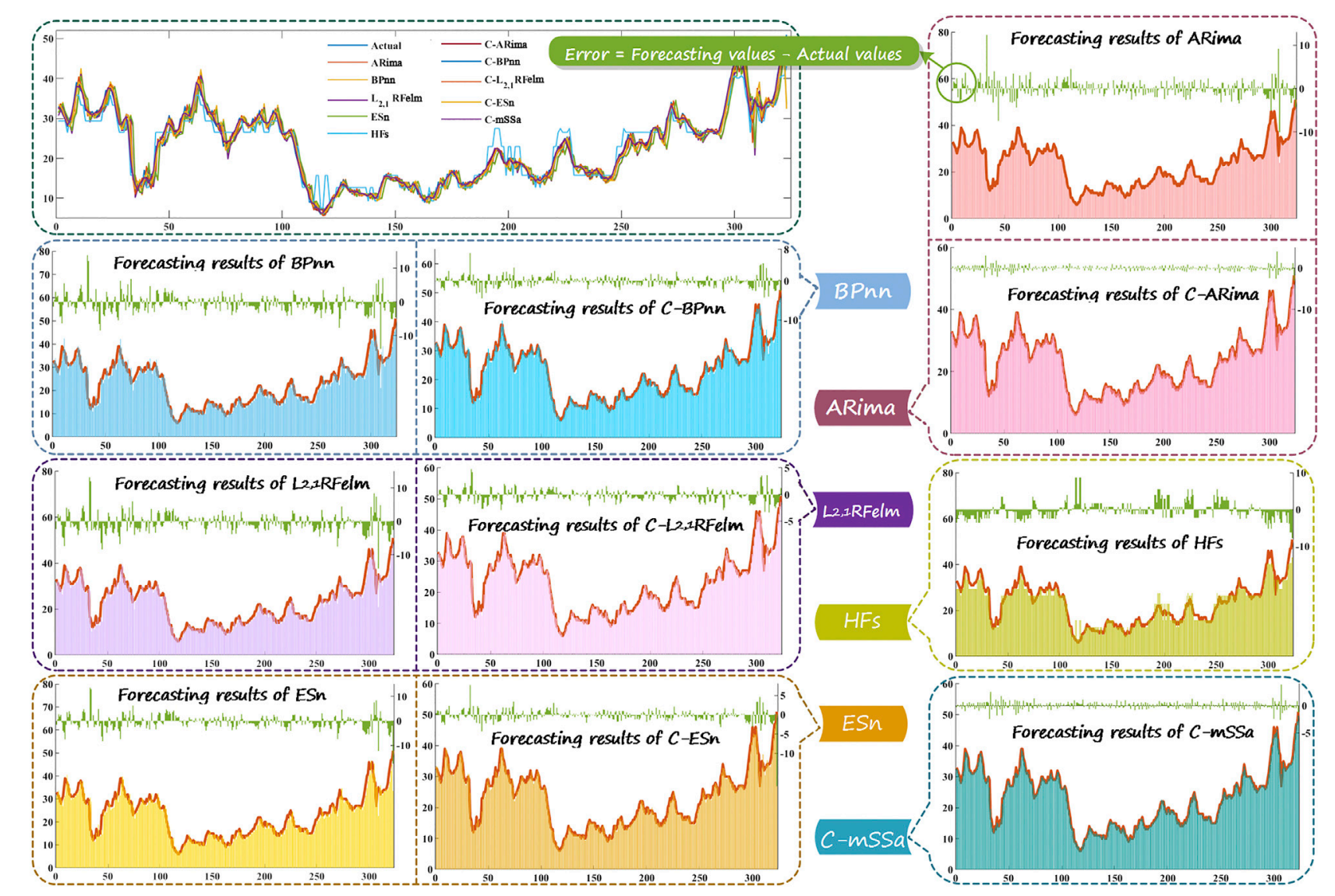

FIGURE 2 | The forecasting results of the different models, where "C-" represents the forecasting models combined with cEEMDan.

$\hat{Y}_{m},(m=1,2, \ldots, M)$, and the weight coefficients of each forecasting result are $\omega_{1}, \omega_{2}, \ldots, \omega_{M}$, then the combined system can be expressed in mathematical form as

$$
\left\{\begin{array}{l}
\mathbf{F}=\sum_{m=1}^{M} \omega_{m} \hat{Y}_{m}, \\
\sum_{m=1}^{M} \Omega_{m}=1,
\end{array}\right.
$$

here $\mathbf{F}$ is the final forecasting result.

The main steps of this proposed system are listed as follows, and the flowchart of this study is described in Figure 1.

Pre-processing of original data. Since the original series are fluctuating, it is difficult to analyze its features. Therefore, the strategy of "decomposition and ensemble" is utilized to distinguish different characteristics and noise in the original series. And then, the noise is filtered out to reconstruct a more stable series. The parameters of this method are shown in Table 5.

Forecasting by individual models. Since the features hidden in the series are not certain, three types of methods were used to analyze the series and implement forecasting. These methods contain a traditional statistical model (ARima), a hesitant fuzzy time series forecasting model, and machine learning models (BPnn, $\ell_{2,1}$ RFelm, ESn). In the three machine learning models, BPnn and $\ell_{2,1}$ RFelm have the same network structure but different solving strategies, BPnn and ESn have different network structures but the same solving strategy, and $\ell_{2,1}$ RFelm and ESn have different network structures and solving strategies.

Construction of the combined system. In order to obtain more accurate forecasting results, use mSSa to conduce the optimal combined weights of the individual models. More specifically, take the predicted values obtained by each individual model as input and the true concentration values as output to form a training set. Then, the optimization algorithm is trained based on this set and finally obtains the optimal weight vector. Afterward, the forecasting results of such individual models are combined together by using optimal weight to obtain the final forecasting value.

\section{EMPIRICAL ANALYSIS}

In this study, the concentration of $\mathrm{PM}_{2.5}$ is forecast by the proposed combined system. This section mainly introduces the experimental process and analyzes the forecasting results.

\subsection{Data Description}

Three $\mathrm{PM}_{2.5}$ concentration data sets collected from the Pearl River Delta (PRD) region in China are selected as illustrative examples to verify the effectiveness of the proposed combined prediction system, including Guangzhou (GZ), Shenzhen (SZ), 


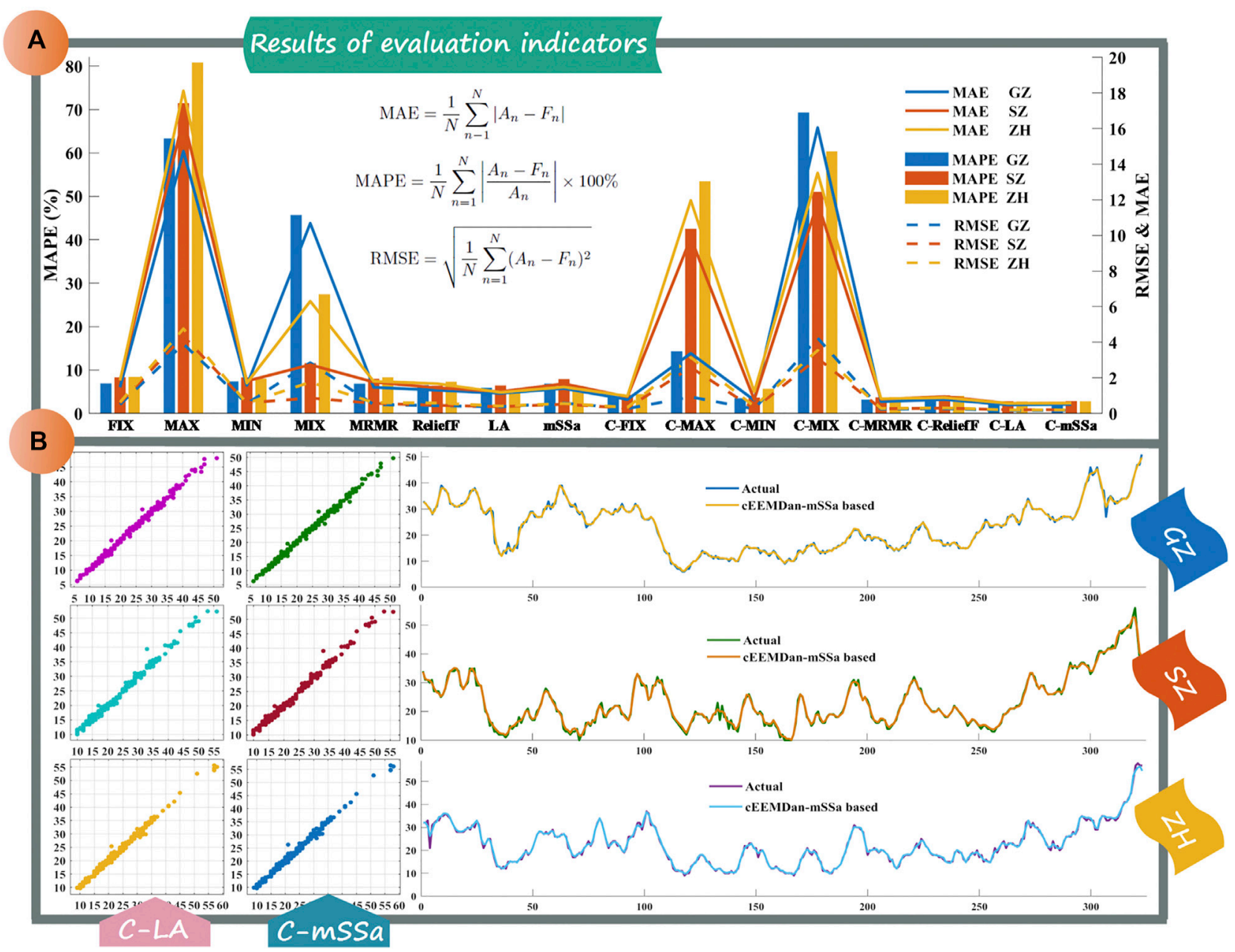

FIGURE 3 | The forecasting results of the different combined methods. (A) is the results of evaluation indicators of three study cities. (B) is the forecasting results of C-LA and C-mSSa, where "C-" represents the cEEMDan.

and Zhuhai $(\mathrm{ZH})$. There are few missing data in these series, and the moving median method with a window length of 10 is used to fill in the missing data. Some statistical indicators for these three data sets are presented in Table 4. Considering the availability of data, the hourly concentrations were collected from 2020.04.29 to 2020.09.25, and these data were divided twice. In the forecasting module, the original data sets were divided into training sets and test sets, and the train to test ratio of each study city is $\operatorname{Tr}_{1}: T e_{1}=7: 3$. And in the combination module, $T e_{1}$ was divided into training set $\operatorname{Tr}_{2}$ and test set $T e_{2}$, the division ratio is $7: 3$.

\subsection{Evaluation Metrics}

In previous studies, numerous metrics have been utilized to evaluate model performance. To scientifically assess the proposed system, three metrics are selected as evaluation criteria, including two scale-dependent indicators and a percentage indicator. Details are as follows.

\subsubsection{Scale-dependent Indicators}

The unit of this type of indicator is the same as the unit of original data, so it can not be used to compare two series with different units. Two commonly used scale-dependent measures are Mean absolute error and Root mean squared error, they are based on absolute errors and squared errors, respectively (Hyndman and Athanasopoulos, 2018).

\section{A. Mean Absolute Error}

The mean absolute error (MAE) is a commonly used indicator to evaluate the deviation between forecast values and true values (Khair et al., 2017):

$$
\text { MAE }=\frac{1}{N} \sum_{n-1}^{N}\left|A_{n}-F_{n}\right|,
$$

where $N$ is the sample size, $A_{n}$ represents the actual value of $n$-th sample, and $F_{n}$ indicates the $n$-th forecast value. This metric can avoid the cancellation of the positive and negative 
predicted errors. The lower the value of MAE, the better the model is. $\mathrm{MAE}=0$ indicates that there is no error in the forecasting.

\section{B. Root Mean Squared Error}

The root mean squared error (RMSE) is a commonly used measure of the forecasting results of machine learning models. Its equation is shown in (Eq. 17) (Wang Y. et al., 2021)

$$
\mathbf{R M S E}=\sqrt{\frac{1}{N} \sum_{n=1}^{N}\left(A_{n}-F_{n}\right)^{2} .}
$$

Same to the MAE, the lower the value of RMSE, the better the prediction. But RMSE is more sensitive to extreme values. Therefore, if the difference between RMSE and MAE is large, the greater the possibility of large errors existing in forecasting.

\subsubsection{Percentage Indicator}

The frequently used percentage indicator is the mean absolute percentage error (MAPE). It is often used in practice since it is a very intuitive explanation in terms of relative error and is unitfree. Its equation is shown as follows (Khair et al., 2017):

$$
\text { MAPE }=\frac{1}{N} \sum_{n=1}^{N} \frac{A_{n}-F_{n}}{A_{n}} \times 100 \% .
$$

Compared to MAE, this indicator is normalized by actual value, and useful when the size or size of a prediction variable is significant in evaluating the accuracy of forecasting (Khair et al., 2017). However, when there is 0 in the actual value, this indicator can not be used. MAPE $=0 \%$ indicates a perfect model, while MAPE $=100 \%$ indicates a poor model.

\subsection{Parameter Settings}

Different parameters of the model will lead to different results, so the analysis of the predicted results should be based on the parameters used. The model parameters used in this paper are shown in Table 5. For ARima, the optimal lag order, the optimal degree of difference, and the optimal order of the moving average are determined based on the Akaike Information Criterion (AIC). And all the empirical experiments are implemented on MATLAB $\mathrm{R} 2020 \mathrm{a}$, run on the Windows 10 professional operating system.

\subsection{Experiments and Results Analysis}

In this study, three comparisons are implemented based on the data from GZ, SZ, and $\mathrm{ZH}$ in China. The first comparison is implemented to verify the effectiveness of the data decomposition strategy, the second comparison compares the different combination methods, and the last comparison compares the individual forecasting methods with the combined forecasting system. The forecasting performance lists in Table 6 and the specific results are analyzed as follows.

\subsubsection{Comparison I}

This comparison is set to compare the forecasting accuracy between the models combining the cEEMDan and models without combining cEEMDan. The comparisons are divided into two categories, one for individual models and one for the combined system. The first category contains comparisons of ARiam vs. C-ARima, BPnn vs. C-BPnn, $\ell_{2,1}$ RFelm vs. C$\ell_{2,1}$ RFelm, and ESn vs. C-ESn. Here, the hesitant fuzzy time series forecasting method has fuzzed the original series and constructed a transition matrix based on the fuzzy logic relationship group to forecast pollution concentration. These operations have compressed and filtered the information of the original series, so the hesitant fuzzy time series forecasting experiment based on the composed data is no longer carried out. The second category contains comparisons of FIX vs. C-FIX, MAX vs. C-MAX, MIN vs. C-MIN, MRMR vs. C-MRMR, ReliefF vs. C-ReliefF, LA vs. C-LA, and mSSa vs. C-mSSa.

1) From the results in Table 6 (a1) and (a2), it can be found that the forecasts based on the decomposed data are more accurate than based on the original data. Take the results from Guangzhou as an example. The maximum MAPE of the forecasts based on decomposed data $\left(\mathrm{MAPE}_{G Z}^{C-B P n n}=5.8968 \%\right)$ is lower than the minimum MAPE of the forecasts based on the original data $\left(\mathrm{MAPE}_{\mathrm{GZ}}^{\text {ARima }}=7.4792 \%\right)$. And the MAE values of the forecasts based on the original data are all greater than $1.1\left(\mathbf{M A E}_{\mathbf{G Z}}>\mathbf{1 . 1}\right)$, but the MAE values of the forecasts based on the decomposed data are all less than $1.1\left(\mathrm{MAE}_{G Z}^{C}<\mathbf{1 . 1}\right)$, especially the $\left(\mathrm{MAE}_{G Z}^{C-A R i m a}<\mathbf{0 . 5}\right)$, which is the best performance among all the forecasting models. The value of RMSE also shows the same result. The values of RMSE for the forecasts based on the original data are all greater than the values of RMSE for the forecasts based on the decomposed data $\left(\mathrm{RMSE}_{G Z}>\mathrm{RMSE}_{G Z}^{C}\right)$, which indicates that the forecasting values based on the decomposed data are closer to the true values. The subfigures in Figure 2 show the predicted results of these models.

2) The strategy of "decomposition and ensemble" to remove noise contributes to improving the forecasting accuracy. The figures in Table 6 (b1) and (b2) show the forecasting results of combined systems. Take $\mathrm{ZH}$ as an example, the values of the indicators of the mSSa combination method are (1.1772, 1.6712,

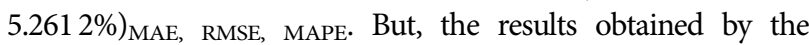
proposed cEEMDan-mSSa based method are (0.5642, 0.7750,

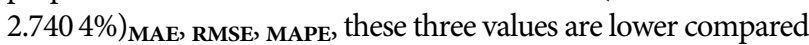
to the index results of $\mathrm{mSSa}$ based combined method. The same relationship can be found in the indicator results for GZ and SZ.

Then, by comparing the remaining figures, it can be found that the values of indicators for systems without combining data decomposition strategy are smaller than the values of combining data decomposition strategy except for the MIX combined method. Take GZ as an example, all the values of MAE are greater than 1 of the method without combining cEEMDan $\left(\mathrm{MAE}_{G Z}>1\right)$, but the values of these indicators are less than 1 for the method combining cEEMDan except for MAX and MIX combined methods $\left(\mathbf{M A E}_{G Z}^{C}<1\right)$. So as the values of RMSE and MAPE, the figures for the methods without combining cEEMDan are greater than the figures for methods combining cEEMDan. Therefore, it can be considered that no matter which combination method, the forecasting based on the decomposed data is more accurate. 
Remark: Through the comparisons between the models combining the cEEMDan and models without combining cEEMDan, what can be found is that the data decomposition strategy can effectively improve the prediction ability of the model.

\subsubsection{Comparison II}

This comparison is set to compare the combination methods. These methods contain four numerical methods (FIX ${ }^{1}, \mathrm{MAX}^{2}$, $\mathrm{MIN}^{3}, \mathrm{MIX}^{4}$ ), two feature selection methods (MRMR, ReliefF), and two optimization algorithms, the Lichtenberg algorithm (LA) and mSSa. The results in Table 6 (b1) and (b2), and Figure 3 demonstrate that after data decomposition, the forecasting accuracy is improved. Moreover, the proposed combined model performance is best. The detailed analyses are as follows.

1) The multi-objective optimization method is the best weighting method. For the results in Table 6 (b1), it can be seen that the indicators' values of mSSa are the smallest. Take GZ as an example, the indicators' values of mSSa are $(1.1232,1.5157,5.8992 \%)_{\text {MAE}}$, RMSE, MAPE, the minimum indicators' values of the numerical methods are $\mathrm{MAE}_{G Z}^{F I X}=1.4641, \mathrm{RMSE}_{G Z}^{F I X}=2.0242, \mathrm{MAPE}_{G Z}^{F I X}=$ 6.8942 , and the minimum indicators' values of the feature selected methods are $\mathrm{MAE}_{G Z}^{\text {ReliefF }}=1.2977, \mathrm{RMSE}_{G Z}^{\text {ReliefF }}=1.7443$, $\mathrm{MAPE}_{G Z}^{\text {ReliefF }}=6.4876$. Based on these indicators' values, it can be seen that the mSSa method has the best forecasting results. So as the results in $\mathrm{SZ}$ and $\mathrm{ZH}$, the indicators' values obtained by $\mathrm{mSSa}$ method are smaller than the value of other methods.

2) Check the results in Table 6 (b2), take $S Z$ as an example, the MAE of numerical methods are (0.9307, 9.8564,0.7709, 11.8266) $)_{\text {FIX }}$, MAX, MIN, MIX, MAE of feature selected methods are $(0.7951,0.9448)_{\text {MRMR, ReliefF, and for the }}$ optimization methods are $(0.5837,0.5670)_{\text {LA }}$, mssa. And $\min (\mathrm{MAE})=\mathbf{M A E}_{S Z}^{C-m S S a}=0.5670$. The same result can be obtained in $\mathrm{GZ}$ and $\mathrm{ZH}$. Based on the results shown in the tables, it can be considered that the mSSa optimization algorithm is optimal as a weighting method.

3) The forecasts of the proposed combined system are more accurate than the mSSa based system. As the forecasting results shown in Table 6 (b1) and (b2), the MAE values of the proposed combined system in the three study cities are MAE $^{\boldsymbol{C - m S S a}}=(0.4776,0.5670,0.5642)_{\mathbf{G Z}, \mathbf{S Z}, \mathbf{Z H}}$, these values are less than 0.6 , but the MAE values of the system based on the original data are greater than 1.1 for three study cites $\left(\mathbf{M A E}^{\mathbf{m S S a}}=(1.1232,1.2227,1.1772)_{\mathbf{G Z}, \mathbf{S Z}, \mathbf{Z H}}\right)$. Moreover,

${ }^{1}$ FIX represents a weighting method with fixed weights, and the weight of each forecasting model is 0.2 .

${ }^{2}$ MAX represents the method of using the maximum forecasting error to assign weights, and the weight of each forecasting model is the reciprocal of the maximum forecasting error obtained by each model in the training set.

${ }^{3}$ MIN is opposite to MAX, using the minimum value of the forecasting error is used as the basis for weighting, the weight of wach methode is caluculated as $w_{i}=e_{i} / \sum_{i=1}^{5} e_{i}, e_{i}=1 / m e_{i}, i=1, \ldots, 5$, where $m e_{i}$ represents minimum error of $i$-th model.

${ }^{4}$ For MIX weighting method, the weight of each model is obtained by following equation: $w_{i}=\operatorname{mean}\left(\left|e_{i n}\right| / A_{n}\right), i=1, \ldots, 5 ; n=1 . \cdots, N$, here the $e_{i n}$ is forecasting errors of $i$-th model, $A_{n}$ is the actual value of $\mathrm{PM}_{2.5}$ concentration. the MAPE values of the proposed system are MAPE ${ }^{\boldsymbol{C}-\boldsymbol{m S S a}}=$ $(2.3576 \%, 2.7879 \%, 2.7404 \%)_{\mathbf{G Z}, \mathbf{S Z}, \mathbf{Z H}}$, compared to the mSSa-based system they are improved by $(60.04,56.65$, $47.91 \%)_{\mathbf{G Z}, \mathbf{S Z}, \mathbf{Z H}}{ }^{5}$. Since the smaller the values of the three metrics, the better the forecasting. Therefore, the results of these metrics indicate that the proposed combined system is performing better than the other system. The same conclusion can be drawn from the values of RMSE.

Remark: The optimization algorithm combination methods are performing better than the other combination methods, especially better than the numerical combination methods. The weights determined by the numerical methods only consider part of the samples, so when the data fluctuates greatly, this type of method cannot get good forecasting results. And the weights determined by the feature selection methods and the optimization algorithms consider all the samples, including samples with large fluctuations, so the impact of large fluctuations can be reduced during the forecasting process.

\subsubsection{Comparison III}

This experiment compares the forecasting performance of the individual forecasting models and the combined forecasting system. The proposed forecasting system performs better than the individual forecasting models. Almost all the indicators' values in the Table 6 (b1) and (b2) are smaller than those in the Table 6 (a1) and (a2), except for the MAX combination method and MIX combination method. Based on the data of SZ, the $\min \left(\mathbf{M A P E}_{S Z}\right)=\mathbf{M A P E}_{S Z}^{C-A R i m a}=4.0504$, but this value is still greater than the MAPE $E_{S Z}^{C-m S S a}=2.7879 \%$. The results of the other two metrics of $S Z$ also show the same relationship. The min $\left(M_{A E}\right)$ and $\min \left(\mathrm{RMSE}_{S Z}\right)$ are $(0.5670,0.8101)$, and all are obtained by the proposed forecasting system. These results indicate that the proposed combined forecasting system outperforms the individual forecasting models. The metric results of $\mathrm{ZH}$ can also draw the same conclusion as SZ. The results in GZ are a little different. The $\min \left(\mathrm{RMSE}_{G Z}\right)=$ $\mathrm{RMSE}_{G Z}^{C-A R i m a}=0.6495$, and $\mathrm{RMSE}_{G Z}^{C-m S S a}=0.6526$, which is only 0.0031 different from the result of C-ARima. Therefore, the performance of the combined forecasting system can be regarded as better than the performance of the individual models.

In summary, the following conclusions can be drawn. The data decomposition strategy can significantly improve forecasting accuracy. These experimental results show that the forecasting results of all methods combined with cEEMDan, except MIX, are more accurate than the methods not combined with cEEMDan. In addition, the mSSa method has the best forecasting results among these combined methods, thus proving the forecasting performance of the proposed system is best.

Remark: For forecasting, data preprocessing is important. In this study, a powerful data decomposition strategy was used to decompose the original data series, and then discarded the noise component of the series. This processing improves the accuracy of the

${ }^{5}$ The improved percentage is calculated as follows: $\mathbf{P}_{\text {metric }}=\left(V_{m}^{\text {Model } 1}-V_{m}^{\text {Model } 2}\right) / V_{m}^{\text {Model1 }}$. Such as the improved percentage of GZ's MAPE is $((5.8992-2.3576) / 5.8992) \times 100 \%$. 
TABLE 7 | DM test results of different models.

\begin{tabular}{|c|c|c|c|c|c|c|c|}
\hline & \multicolumn{3}{|c|}{ Based on the original data } & \multirow[t]{2}{*}{-} & \multicolumn{3}{|c|}{ Based on the decomposed data } \\
\hline & GZ & SZ & $\mathbf{Z H}$ & & GZ & Sz & $\mathbf{Z H}$ \\
\hline \multicolumn{8}{|c|}{ (a) Forecasting models } \\
\hline ARima & $5.0977^{\star}$ & $8.6243^{\star}$ & $6.3830^{\star}$ & C-ARima & $1.8092^{\star \star}$ & $1.1831^{\star \star \star}$ & $1.2560^{\star \star \star}$ \\
\hline BPnn & $6.8607^{\star}$ & $10.4830^{*}$ & $8.5372^{\star}$ & C-BPnn & $7.2128^{*}$ & $8.3443^{\star}$ & $9.5224^{\star}$ \\
\hline$\ell_{2,1}$ RFelm & $7.0826^{\star}$ & $9.5058^{\star}$ & $9.0820^{*}$ & $\mathrm{C}-\ell_{2,1}$ RFelm & $6.6667^{\star}$ & $7.9443^{\star}$ & $8.2492^{*}$ \\
\hline ESn & $5.9861^{\star}$ & $9.2922^{*}$ & $8.1848^{\star}$ & C-ESn & $2.1994^{\star \star}$ & $7.8597^{\star}$ & $2.5456^{\star \star}$ \\
\hline HFs & $8.5816^{\star}$ & $11.6504^{*}$ & $5.2527^{\star}$ & - & - & - & - \\
\hline \multicolumn{8}{|c|}{ (b) Combined systems } \\
\hline FIX & $7.2134^{\star}$ & $9.0465^{\star}$ & $7.7204^{\star}$ & C-FIX & $4.5435^{\star}$ & $5.3059^{\star}$ & $11.5207^{\star}$ \\
\hline MAX & $22.7947^{\star}$ & $21.5754^{\star}$ & $20.7482^{*}$ & C-MAX & $16.9403^{\star}$ & $21.2405^{\star}$ & $14.4119^{*}$ \\
\hline $\mathrm{MIN}$ & $6.3787^{\star}$ & $6.1052^{\star}$ & $5.9078^{\star}$ & C-MIN & $5.1935^{\star}$ & $4.8392^{\star}$ & $12.5494^{\star}$ \\
\hline MIX & $21.4592^{\star}$ & $10.6720^{\star}$ & $14.4818^{*}$ & C-MIX & $23.2960^{\star}$ & $21.4463^{\star}$ & $16.1001^{\star}$ \\
\hline MRMR & $6.7769^{\star}$ & $9.0465^{\star}$ & $8.4095^{\star}$ & C-MRMR & $3.9308^{*}$ & $4.1445^{\star}$ & $11.9718^{\star}$ \\
\hline ReliefF & $8.2291^{\star}$ & $8.4603^{\star}$ & $4.9277^{\star}$ & C-ReliefF & $5.2545^{\star}$ & $5.4203^{\star}$ & $10.6837^{\star}$ \\
\hline LA & $2.2041^{\star \star}$ & $9.7650^{\star}$ & $5.5175^{\star}$ & C-LA & $1.6563^{\star \star}$ & $2.2618^{\star \star}$ & $0.5763^{\star \star \star}$ \\
\hline $\mathrm{mSSa}$ & $6.7769^{\star}$ & $8.6787^{\star}$ & $5.8400^{\star}$ & C-mSSa & - & - & - \\
\hline
\end{tabular}

TABLE 8 | Results of the model stability test.

\begin{tabular}{|c|c|c|c|c|c|c|c|}
\hline & \multicolumn{3}{|c|}{ Based on the original data } & \multirow[t]{2}{*}{-} & \multicolumn{3}{|c|}{ Based on the decomposed data } \\
\hline & GZ & sz & $\mathbf{Z H}$ & & GZ & SZ & $\mathbf{Z H}$ \\
\hline \multicolumn{8}{|c|}{ (a) Forecasting models } \\
\hline ARima & 0.9664 & 0.9658 & 0.8981 & C-ARima & 0.9841 & 0.9881 & 0.9721 \\
\hline BPnn & 0.9902 & 0.9841 & 0.9915 & C-BPnn & 0.9971 & 0.9936 & 0.9644 \\
\hline$\ell_{2,1}$ RFelm & 0.9643 & 0.9943 & 0.9262 & $\mathrm{C}-\ell_{2,1}$ RFelm & 0.9979 & 0.9577 & 0.9935 \\
\hline ESn & 0.9288 & 0.9126 & 0.8376 & C-ESn & 0.9564 & 0.9932 & 0.9144 \\
\hline \multicolumn{8}{|c|}{ (b) Combined systems } \\
\hline FIX & 0.8996 & 0.8947 & 0.8625 & C-FIX & 0.9318 & 0.9521 & 0.9420 \\
\hline MAX & 0.1179 & 0.0734 & 0.0329 & C-MAX & 0.7009 & 0.3225 & 0.2122 \\
\hline MIN & 0.9350 & 0.9234 & 0.8794 & C-MIN & 0.9909 & 0.9863 & 0.9995 \\
\hline MIX & 0.2701 & 0.7733 & 0.4789 & C-MIX & 0.0852 & 0.2325 & 0.1557 \\
\hline MRMR & 0.9254 & 0.9179 & 0.8755 & C-MRMR & 0.9526 & 0.9676 & 0.9545 \\
\hline
\end{tabular}

"C-" represents the forecasting models combined with cEEMDan, that is the forecasting models based on the decomposed data.

Indicates that the stability test has not been performed. Since HFs have compressed the original series, the forecasting based on the decomposed data has not been performed. And the

C-mSSa is the system proposed in this paper, so the stability test has not been performed on itself.

forecasting, and this conclusion is reached in two experiments. For combination, the multi-objective optimization method works better, and the numerical methods are the worst, and the performance is unstable. When the results of other methods become better, the numerical method performs worse.

\section{TEST OF FORECASTING SYSTEM}

In order to verify the significance and stability of the proposed forecasting system, the Diebold-Mariano test (DM) (Francis and Roberto, 1995) and the variance ratio (VR) are introduced in this study. The related details and results are described in this section.

\subsection{Diebold-Mariano Test}

$\mathrm{DM}$ is a hypothesis testing method to analyze the difference in prediction accuracy. According to the constructed DM statistics, it can be judged whether the difference of the prediction method is significant. In this test, the null hypothesis $\left(H_{0}\right)$ and the alternative hypothesis $\left(H_{1}\right)$ are as follows:

$$
\begin{aligned}
& \boldsymbol{H}_{0}: \overline{\overline{\boldsymbol{E}}}\left[\tilde{\mathcal{L}}\left(\breve{\boldsymbol{\delta}}_{1}^{t}\right)\right]=\overline{\overline{\boldsymbol{E}}}\left[\tilde{\mathcal{L}}\left(\breve{\boldsymbol{\delta}}_{2}^{t}\right)\right] \\
& \boldsymbol{H}_{1}: \overline{\overline{\boldsymbol{E}}}\left[\tilde{\mathcal{L}}\left(\breve{\boldsymbol{\delta}}_{1}^{t}\right)\right] \neq \overline{\bar{E}}\left[\tilde{\mathcal{L}}\left(\breve{\boldsymbol{\delta}}_{2}^{t}\right)\right]
\end{aligned}
$$


here $\breve{\delta}_{1}^{t}$ and $\breve{\delta}_{2}^{t}$ represent the forecasting errors of forecasting model 1 and forecasting model 2 at $t$-th, $\mathcal{L}(\cdot)$ represents the loss function. Then, the DM statistic is constructed as follows (Huang et al., 2021):

$$
\mathrm{DM}=\frac{\sum_{t=1}^{n}\left[\tilde{\mathcal{L}}\left(\breve{\delta}_{1}^{t}\right)-\tilde{\mathcal{L}}\left(\breve{\delta}_{2}^{t}\right)\right] / n}{\sqrt{S^{2} / n}}
$$

where $S^{2}$ denotes the variance estimation of $\breve{\delta}_{1}^{t}-\breve{\delta}_{2}^{t}$.

Given a certain significance level $\alpha$, the critical value $Z_{\alpha / 2}$ can get, if the absolute value of DM statistic is greater than the $Z_{\alpha / 2}$, the null hypothesis $\boldsymbol{H}_{\mathbf{0}}$ is rejected, and the result that two forecasting methods have significant differences.

Table 7 gives the DM test results of different forecasting models. This study compares 24 forecasting models or systems with the proposed system. Compared with the forecasting model without cEEMDan, the proposed forecasting system is significantly better, since the values of DM statistic are greater than the critical value of $1 \%$ significance level. After combined with cEEMDan, the forecasting ability of individual forecasting models has been improved, but the DM test results show that their predictive ability is still inferior to the proposed forecasting system, since the lowest value of DM test is between the critical value of $10 \%$ significance level and the critical value of $15 \%$ significance level. The DM values of Table 7 (b) also show that the proposed forecasting system is significantly superior than the other combined forecasting system, especially the system without data decomposition strategy.

\subsubsection{Stability Test}

In order to validate the stability of models, the variance ratio (Vr) is introduced. $\mathbf{V r}$ combines the variances of the forecasting value and the true value to illustrate the stability of the forecasting model. The greater the value of $\mathrm{Vr}$, the higher the forecasting stability of the method (Huang et al., 2021).

$$
\boldsymbol{V r}=\min \left(\operatorname{Var}_{\text {forecasting }} / \operatorname{Var}_{\text {actual }}, \operatorname{Var}_{\text {actual }} / \operatorname{Var}_{\text {forecasting }}\right) \text {, }
$$

here, $\boldsymbol{V a r}_{\text {forecasting }}$ and $\boldsymbol{V a r}_{\text {actual }}$ are the variances of the forecasting values and actual values.

The Vr results are shown in Table 8. The Vr values of the proposed system in the three cities are (0.9861, 0.9986, $0.9859)_{\mathrm{GZ,SZ,ZH}}$. Although the $\mathrm{Vr}$ values of the proposed system are not the largest among all forecasting models and systems, these three values are all greater than 0.98 , while the $\mathrm{Vr}$ values of most other forecasting models and systems are less than 0.98 , indicating that the proposed forecasting system is relatively stable. Combined with the results of the forecasting evaluation metric shown in section 3, it shows that the proposed forecasting system has high prediction accuracy and relatively high stability.

\section{CONCLUSION}

Based on the multi-objective optimization algorithm and data decomposition strategy, an effective combined forecasting system is proposed to forecast the $\mathrm{PM}_{2.5}$ concentration from Guangzhou, Shenzhen, and Zhuhai in China. The proposed system mainly contains three modules, the data preprocessing module, the individual model forecasting module, and the combination forecasting module. In the first module, the strategy of "decomposition and ensemble" is applied to remove the noise in the original series. In the individual model forecasting module, ARima, BPnn, $\ell_{2,1}$ RFelm, ESn, and HFs are applied to forecast $\mathrm{PM}_{2.5}$ concentration respectively. These five models are from different kinds of forecasting models and are used to analyze different features in the $\mathrm{PM}_{2.5}$ concentration series. ARima is a classical traditional statistical forecasting method; BPnn, $\ell_{2,1}$ RFelm, and ESn are neural networks with different characteristics; hesitant fuzzy time series model is a fuzzy-based forecasting model. By comparing eight weighting methods from three categories, the best combination method is found as a multi-objective optimization weighting method.

The developed combined forecasting system has been successfully applied in $\mathrm{PM}_{2.5}$ concentration forecasting. Based on the forecasting evaluation indicators, the forecasting performance of the proposed system is validated. Specifically, compared the models forecasting results based on data before and after the preprocessing of cEEMDan in Comparison I. In Comparison II, compare the system employing diverse combination methods. Compere between the individual models and the combined models in Comparison III. After these comparative experiments, it can be observed that the MAE and MAPE values of the proposed system are always lower than the values of individual models and other combination methods. For RMSE in Guangzhou, the value of the proposed system is slightly higher than the minimum RMSE value, but overall, the forecasting performance of the proposed system is still the best. Therefore, the proposed combined forecasting system, which combines different types of individual forecasting models, has high practical application potential in air pollution concentration forecasting.

\section{DATA AVAILABILITY STATEMENT}

Publicly available datasets were analyzed in this study. This data can be found here: http://www.mep.gov.cn/.

\section{AUTHOR CONTRIBUTIONS}

LB: Conceptualization, Methodology, Software, Writing-Original draft preparation. HL: Conceptualization, Software, Validation. BZ: Conceptualization, Supervision, Writing-Reviewing and Editing. XH: Conceptualization, Writing-Review and Editing, Data curation.

\section{FUNDING}

This work was supported by Major Program of National Social Science Foundation of China (Grant No. 17ZDA093). 


\section{REFERENCES}

Ariyo, A. A., Adewumi, A. O., and Ayo, C. K. (2014). "Stock price Prediction Using the Arima Model," in 2014 UKSim-AMSS 16th International Conference on Computer Modelling and Simulation (Cambridge, UK: IEEE), 106-112. doi:10. 1109/uksim.2014.67

Benvenuto, D., Giovanetti, M., Vassallo, L., Angeletti, S., and Ciccozzi, M. (2020). Application of the Arima Model on the Covid-2019 Epidemic Dataset. Data in brief 29, 105340. doi:10.1016/j.dib.2020.105340

Bisht, K., and Kumar, S. (2016). Fuzzy Time Series Forecasting Method Based on Hesitant Fuzzy Sets. Expert Syst. Appl. 64, 557-568. doi:10.1016/j.eswa.2016. 07.044

Cheng, S.-H., Chen, S.-M., and Jian, W.-S. (2016). Fuzzy Time Series Forecasting Based on Fuzzy Logical Relationships and Similarity Measures. Inf. Sci. 327, 272-287. doi:10.1016/j.ins.2015.08.024

Cheng, X., Liu, Y., Xu, X., You, W., Zang, Z., Gao, L., et al. (2019). Lidar Data Assimilation Method Based on Crtm and Wrf-Chem Models and its Application in pm2.5 Forecasts in Beijing. Sci. Total Environ. 682, 541-552. doi:10.1016/j.scitotenv.2019.05.186

Dèdelè, A., and Miškinytè, A. (2019). Seasonal and Site-specific Variation in Particulate Matter Pollution in lithuania. Atmos. Pollut. Res. 10, 768-775. doi:10.1016/j.apr.2018.12.004

Francis, X., and Roberto, S. (1995). Comparing Predictive Accuracy. J. Business Econ. Stat. 13, 134. doi:10.1080/07350015.1995.10524599

Gavirangaswamy, V. B., Gupta, G., Gupta, A., and Agrawal, R. (2013). “Assessment of Arima-Based Prediction Techniques for Road-Traffic Volume," in Proceedings of the fifth international conference on management of emergent digital EcoSystems, 246-251. doi:10.1145/2536146.2536176

Glencross, D. A., Ho, T.-R., Camiña, N., Hawrylowicz, C. M., and Pfeffer, P. E. (2020). Air Pollution and its Effects on the Immune System. Free Radic. Biol. Med. 151, 56-68. doi:10.1016/j.freeradbiomed.2020.01.179

Goyal, P., Chan, A. T., and Jaiswal, N. (2006). Statistical Models for the Prediction of Respirable Suspended Particulate Matter in Urban Cities. Atmos. Environ. 40, 2068-2077. doi:10.1016/j.atmosenv.2005.11.041

Grennfelt, P., Engleryd, A., Forsius, M., Hov, Ø., Rodhe, H., and Cowling, E. (2020). Acid Rain and Air Pollution: 50 Years of Progress in Environmental Science and Policy. Ambio 49, 849-864. doi:10.1007/s13280-019-01244-4

Gündüz, D., de Kerret, P., Sidiropoulos, N. D., Gesbert, D., Murthy, C. R., and van der Schaar, M. (2019). Machine Learning in the Air. IEEE J. Selected Areas Commun. 37, 2184-2199.

Haimes, Y. Y., Hall, W. A., and Freedman, H. T. (2011). Multiobjective Optimization in Water Resources Systems: The Surrogate worth Trade-Off Method. Amsterdam, Netherlands: Elsevier.

Hecht-Nielsen, R. (1992). “Theory of the Backpropagation Neural Network ${ }^{* *}$ Based on "nonindent" by Robert Hecht-Nielsen, Which Appeared in Proceedings of the International Joint Conference on Neural Networks 1, 593-611, June 1989. 1989 IEEE," in Neural Networks for Perception (Amsterdam, Netherlands: Elsevier), 65-93. doi:10.1016/b978-0-12-741252-8.50010-8

Henrique, B. M., Sobreiro, V. A., and Kimura, H. (2019). Literature Review: Machine Learning Techniques Applied to Financial Market Prediction. Expert Syst. Appl. 124, 226-251. doi:10.1016/j.eswa.2019.01.012

Huang, X., Wang, J., and Huang, B. (2021). Two Novel Hybrid Linear and Nonlinear Models for Wind Speed Forecasting. Energ. Convers. Manage. 238, 114162. doi:10.1016/j.enconman.2021.114162

Hyndman, R. J., and Athanasopoulos, G. (2018). Forecasting: Principles and Practice. Melbourne, Australia: OTexts.

Jaeger, H., and Haas, H. (2004). Harnessing Nonlinearity: Predicting Chaotic Systems and Saving Energy in Wireless Communication. science 304, 78-80. doi:10.1126/science.1091277

Khair, U., Fahmi, H., Hakim, S. A., and Rahim, R. (2017). Forecasting Error Calculation with Mean Absolute Deviation and Mean Absolute Percentage Error. J. Phys. Conf. Ser. 930, 012002. doi:10.1088/1742-6596/930/1/012002

Kurt, A., Gulbagci, B., Karaca, F., and Alagha, O. (2008). An Online Air Pollution Forecasting System Using Neural Networks. Environ. Int. 34, 592-598. doi:10. 1016/j.envint.2007.12.020

Liu, B., Yu, X., Chen, J., and Wang, Q. (2021). Air Pollution Concentration Forecasting Based on Wavelet Transform and Combined Weighting
Forecasting Model. Atmos. Pollut. Res. 12, 101144. doi:10.1016/j.apr.2021. 101144

Liu, H., Xu, Y., and Chen, C. (2019). Improved Pollution Forecasting Hybrid Algorithms Based on the Ensemble Method. Appl. Math. Model. 73, 473-486. doi:10.1016/j.apm.2019.04.032

Lu, W., Chen, X., Pedrycz, W., Liu, X., and Yang, J. (2015). Using Interval Information Granules to Improve Forecasting in Fuzzy Time Series. Int. J. Approximate Reasoning 57, 1-18. doi:10.1016/j.ijar.2014.11.002

Manisalidis, I., Stavropoulou, E., Stavropoulos, A., and Bezirtzoglou, E. (2020). Environmental and Health Impacts of Air Pollution: a Review. Front. Public Health 8, 14. doi:10.3389/fpubh.2020.00014

Mirjalili, S., Gandomi, A. H., Mirjalili, S. Z., Saremi, S., Faris, H., and Mirjalili, S. M. (2017a). Salp Swarm Algorithm: A Bio-Inspired Optimizer for Engineering Design Problems. Adv. Eng. Softw. 114, 163-191. doi:10.1016/j.advengsoft. 2017.07.002

Mirjalili, S., Jangir, P., and Saremi, S. (2017b). Multi-objective Ant Lion Optimizer: a Multi-Objective Optimization Algorithm for Solving Engineering Problems. Appl. Intell. 46, 79-95. doi:10.1007/s10489-016-0825-8

Mousavi, S. S., Goudarzi, G., Sabzalipour, S., Rouzbahani, M. M., and Mobarak Hassan, E. (2021). An Evaluation of Co, Co2, and So2 Emissions during Continuous and Non-continuous Operation in a Gas Refinery Using the Aermod. Environ. Sci. Pollut. Res. 28, 56996-57008. doi:10.1007/s11356-02114493-2

Ngatchou, P., Zarei, A., and El-Sharkawi, A. (2005). "Pareto Multi Objective Optimization," in Proceedings of the 13th International Conference on, Intelligent Systems Application to Power Systems (Arlington, VA, USA: IEEE), 84-91.

Niska, H., Hiltunen, T., Karppinen, A., Ruuskanen, J., and Kolehmainen, M. (2004). Evolving the Neural Network Model for Forecasting Air Pollution Time Series. Eng. Appl. Artif. Intelligence 17, 159-167. doi:10.1016/j.engappai.2004. 02.002

Niu, X., and Wang, J. (2019). A Combined Model Based on Data Preprocessing Strategy and Multi-Objective Optimization Algorithm for Short-Term Wind Speed Forecasting. Appl. Energ. 241, 519-539. doi:10.1016/j.apenergy.2019.03.097

Organization, W. H. (2014). 7 Million Premature Deaths Annually Linked to Air Pollution. Geneve, Switzerland: WHO.

Pai, P.-F., and Lin, C.-S. (2005). A Hybrid Arima and Support Vector Machines Model in Stock price Forecasting. Omega 33, 497-505. doi:10.1016/j.omega. 2004.07.024

Qiao, J., Li, F., Han, H., and Li, W. (2016). Growing echo-state Network with Multiple Subreservoirs. IEEE Trans. Neural Netw. Learn. Syst. 28, 391-404. doi:10.1109/TNNLS.2016.2514275

Rahimi, A., and Recht, B. (2007). "Random Features for Large-Scale Kernel Machines," in Proceedings of the 20th International Conference on Neural Information Processing Systems (New York: Curran Associates Inc.), $1177-1184$.

Singh, S. R. (2007). A Simple Method of Forecasting Based on Fuzzy Time Series. Appl. Math. Comput. 186, 330-339. doi:10.1016/j.amc.2006.07.128

Song, Q., and Chissom, B. S. (1993). Fuzzy Time Series and its Models. Fuzzy sets Syst. 54, 269-277. doi:10.1016/0165-0114(93)90372-o

Torra, V., and Narukawa, Y. (2009). "On Hesitant Fuzzy Sets and Decision," in 2009 IEEE International Conference on Fuzzy Systems (Jeju, South Korea: IEEE), 1378-1382. doi:10.1109/fuzzy.2009.5276884

Torres, M. E., Colominas, M. A., Schlotthauer, G., and Flandrin, P. (2011). "A Complete Ensemble Empirical Mode Decomposition with Adaptive Noise," in 2011 IEEE international conference on acoustics, speech and signal processing (ICASSP) (Prague, Czech Republic: IEEE), 4144-4147. doi:10.1109/icassp.2011. 5947265

Volk, M. J., Lourentzou, I., Mishra, S., Vo, L. T., Zhai, C., and Zhao, H. (2020). Biosystems Design by Machine Learning. ACS Synth. Biol. 9, 1514-1533. doi:10. 1021/acssynbio.0c00129

Wang, J., Du, P., Hao, Y., Ma, X., Niu, T., and Yang, W. (2020a). An Innovative Hybrid Model Based on Outlier Detection and Correction Algorithm and Heuristic Intelligent Optimization Algorithm for Daily Air Quality index Forecasting. J. Environ. Manag. 255, 109855. doi:10.1016/j.jenvman.2019. 109855

Wang, J., Li, H., Wang, Y., and Lu, H. (2021a). A Hesitant Fuzzy Wind Speed Forecasting System with Novel Defuzzification Method and Multi-Objective 
Optimization Algorithm. Expert Syst. Appl. 168, 114364. doi:10.1016/j.eswa. 2020.114364

Wang, J., Li, Q., and Zeng, B. (2021b). Multi-layer Cooperative Combined Forecasting System for Short-Term Wind Speed Forecasting. Sustainable Energ. Tech. Assessments 43, 100946. doi:10.1016/j.seta.2020.100946

Wang, J., Niu, T., Lu, H., Yang, W., and Du, P. (2019). A Novel Framework of Reservoir Computing for Deterministic and Probabilistic Wind Power Forecasting. IEEE Trans. Sustain. Energ. 11, 337-349.

Wang, J., Wang, Y., Li, Z., Li, H., and Yang, H. (2020b). A Combined Framework Based on Data Preprocessing, Neural Networks and Multi-Tracker Optimizer for Wind Speed Prediction. Sustain. Energ. Tech. Assessments 40, 100757. doi:10.1016/j.seta.2020.100757

Wang, J., Yang, W., Du, P., and Niu, T. (2020c). Outlier-robust Hybrid Electricity price Forecasting Model for Electricity Market Management. J. Clean. Prod. 249, 119318. doi:10.1016/j.jclepro.2019.119318

Wang, Y.-H., Yeh, C.-H., Young, H.-W. V., Hu, K., and Lo, M.-T. (2014). On the Computational Complexity of the Empirical Mode Decomposition Algorithm. Physica A: Stat. Mech. its Appl. 400, 159-167. doi:10.1016/j.physa.2014.01.020

Wang, Y., Wang, J., Li, Z., Yang, H., and Li, H. (2021c). Design of a Combined System Based on Two-Stage Data Preprocessing and Multi-Objective Optimization for Wind Speed Prediction. Energy 231, 121125. doi:10.1016/j.energy.2021.121125

Yang, H., Zhu, Z., Li, C., and Li, R. (2020). A Novel Combined Forecasting System for Air Pollutants Concentration Based on Fuzzy Theory and Optimization of Aggregation Weight. Appl. Soft Comput. 87, 105972. doi:10.1016/j.asoc.2019.105972

Zadeh, L. A. (1996). "Fuzzy Sets," in Fuzzy Sets, Fuzzy Logic, and Fuzzy Systems: Selected Papers by Lotfi A Zadeh (Singapore: World Scientific), 394-432. doi:10. 1142/9789814261302_0021
Zhang, L., Lin, J., Qiu, R., Hu, X., Zhang, H., Chen, Q., et al. (2018). Trend Analysis and Forecast of pm2.5 in Fuzhou, china Using the Arima Model. Ecol. Indicators 95, 702-710. doi:10.1016/j.ecolind.2018.08.032

Zhou, S., Liu, X., Liu, Q., Wang, S., Zhu, C., and Yin, J. (2016). Random Fourier Extreme Learning Machine with $\ell_{2,1}$-Norm Regularization. Neurocomputing 174, 143-153. doi:10.1016/j.neucom.2015.03.113

Zhu, S., Lian, X., Liu, H., Hu, J., Wang, Y., and Che, J. (2017). Daily Air Quality index Forecasting with Hybrid Models: A Case in china. Environ. Pollut. 231, 1232-1244. doi:10.1016/j.envpol.2017.08.069

Conflict of Interest: The authors declare that the research was conducted in the absence of any commercial or financial relationships that could be construed as a potential conflict of interest.

Publisher's Note: All claims expressed in this article are solely those of the authors and do not necessarily represent those of their affiliated organizations, or those of the publisher, the editors and the reviewers. Any product that may be evaluated in this article, or claim that may be made by its manufacturer, is not guaranteed or endorsed by the publisher.

Copyright (c) 2022 Bai, Li, Zeng and Huang. This is an open-access article distributed under the terms of the Creative Commons Attribution License (CC BY). The use, distribution or reproduction in other forums is permitted, provided the original author(s) and the copyright owner(s) are credited and that the original publication in this journal is cited, in accordance with accepted academic practice. No use, distribution or reproduction is permitted which does not comply with these terms. 